\title{
Mathematical modeling and optimal intervention strategies of the COVID-19 outbreak
}

\author{
Jayanta Mondal • Subhas Khajanchi*
}

Received: date / Accepted: date

\begin{abstract}
COVID-19 pandemic were recorded on 30 August 2021 in India. To end this ongoing global COVID-19 pandemic, there is an urgent need to implement multiple population-wide policies like social distancing, testing more people and contact tracing. To predict the course of the pandemic and come up with a strategy to control it effectively, a compartmental model has been established. The following six stages of infection are taken into consideration: susceptible $(S)$, asymptomatic infected $(A)$, clinically ill or symptomatic infected $(I)$, quarantine $(Q)$, isolation $(J)$ and recovered $(R)$, collectively termed as SAIQJR. The qualitative behavior of the model and the stability of biologically realistic equilibrium points are investigated in terms of the basic reproduction number. We performed sensitivity analysis with respect to the basic reproduction number and obtained that the disease transmission rate has an impact in mitigating the spread of diseases. Moreover, considering the non-pharmaceutical and pharmaceutical intervention strategies as control functions, an optimal control problem is implemented to mitigate the disease fatality. To reduce the infected individuals and to minimize the cost of the controls, an objective functional has been constructed and solved with the aid of Pontryagin's Maximum Principle. The implementation of optimal control strategy at the start
\end{abstract}

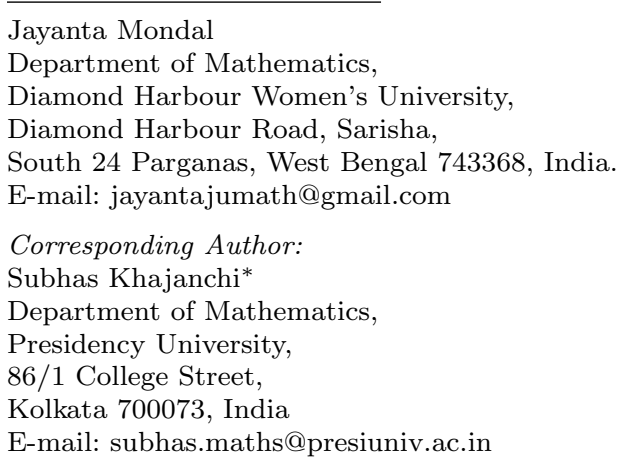


of a pandemic tends to decrease the intensity of epidemic peaks, spreading the maximal impact of an epidemic over an extended time period. Extensive numerical simulations show that the implementation of intervention strategy has an impact in controlling the transmission dynamics of COVID-19 epidemic. Further, our numerical solutions exhibit that the combination of three controls are more influential when compared with the combination of two controls as well as single control. Therefore the implementation of all the three control strategies may help to mitigate novel coronavirus disease transmission at this present epidemic scenario.

Keywords Model calibration · Isolation or hospitalization · Optimal control · Pontryagin maximum principle $\cdot$ Sensitivity analysis

\section{Introduction}

As of August 30, 2021, the COVID-19 pandemic has already crossed 216,303,376 infected cases and more than 4,498,451 deaths throughout the world, becoming the considerable public health problem facing after the Second World War. It has a great impact to forecast the trend of SARS-CoV-2 pandemic circumstances. At this highly advanced stage, it is of utmost priority to reply some questions immediately: when the COVID-19 infection can be entirely removed by proper vaccination or immunization, and what is the interaction among the threshold of vaccination and disease reproduction number $R_{0}$ [1. The SARS-CoV-2 has an important research as it was identified as pandemic by the World Health Organization (WHO) on March 11, 2020 and obtaining a lot of recognition of researchers/scientists in diverse areas of people knowledge.

Dynamical viewpoint is a fascinating outlook to deal with the bio-medical systems. Several literature presents different outlooks regarding dynamical models for an epidemic [2], generally utilized to delineate various infectious diseases. A fascinating and convenient outlook is rely on the population dynamics, where various individuals are employed to indicate the diseases, representing their evolution and interplays. Kermack and McKendrick 3] were one of the pioneers in developing 3 individuals in epidemic dynamic models, namely Susceptible, Infectious and Recovered (SIR). Their mathematical model delineates the course of outbreak exhibiting that it never be terminated necessarily by all populations becoming infectious. Anderson and May [4 modified the wellestablished SIR model developed by Kermack and McKendrick [3, incorporating an additional compartment: exposed class $(E)$. Presently, most of the epidemic model is rely on the susceptible-exposed-infected-removed (SEIR) structure, widely used to delineate various infectious diseases as Hepatitis, HIV, HTLV, Dengue, Typhoid, among others.

The first case of coronavirus was identified at Thrissur district, Kerala in India on January 30, 2020, when a student came from Wuhan, China. Due to the India COVID-19 tracker [5] there are 32,767,824 total confirmed cases, 
31,952,217 recovered people and 438,592 total deaths are recorded in the Republic of India on August 30, 2021. The Govt. of India has declared to continue social distance or to adopt self-quarantine policy in order to ignore community transmission of the novel coronavirus diseases among the population. On 22 March 2020, the Indian Govt. has implemented a "Janata Curfew" during 14-hours while the total number of infected cases became more than 500 individuals. After that the Indian Govt. announced a nationwide lockdown for 21 days starting from 25 March 2020 in order to mitigate the spread of the novel coronavirus transmission. Later on the Indian Govt. increased the nationwide lockdown to slow down the transmission of novel coronavirus pandemic. Due to the limited vaccine for COVID-19 diseases, the people must have to maintain social distance, wearing mask, avoid mass gathering or applying self-isolation to prevent the transmission of novel coronavirus [6]. The nationwide lockdown incorporates prohibition on individuals from stepping out of their houses, stop cultural functions, mass gathering, shutdown of all stores/shops excluding medicine shops, school/colleges, banks, hospitals, nursing home, etc., postponement of all the academic college/university/institution (allowed work from home only), postponement of all the private and public cars and also the closure of all cultural activities, political gathering, sports, entertainments, etc. This epidemic has significantly affected the life of all the people from economically as well as health. According to the report from the World Bank and the Reserve Bank of India (RBI), after the Second World War, this is the first time when the economic growth rate of India and the World as a whole will be diminished by 1.5-2.8\% due to the effect of COVID-19 outbreak. Indeed the expansion of this novel coronavirus outbreak has disrupted the daily life of the people, economic and the health of the people. Thus, this is one of the major issues for all the people how long this situation will continue and when the COVID-19 disease will be under maintained. In order to control the novel coronavirus diseases, the scientists around the world has been trying to improve the vaccine quality such that the disease can be eliminated.

Due to the speedy expansion of novel coronavirus, which lead to necessary beds in medical colleges saturating in many countries [7] virtually many countries executed some form of non-pharmaceutical interventions (NPIs) 6. 8. The aim of these intervention strategies was mainly to "flatten the curve" indicating that a mitigation of the coronavirus outbreak could be attained through a change of behavior in the human [9]. Flaxman et al. 8] quantifies the role of different non-pharmaceutical interventions on the transmission number, $R_{t}$, utilizing real data from the 11 different countries of Europe that were dealing with the novel coronavirus disease during the first wave of infections. An important interpretation is that the close progression of the nonpharmaceutical intervention strategies make it hard to obtain a clear answer as to which non-pharmaceutical intervention strategy has the most impact. Also, non-pharmaceutical intervention strategy take sometime to be effective. Thus, since the real alteration of the expansion happens much later than the intervention dates, the cumulative alteration tends to become aggregated in 
latest possible time window. The outbreak of COVID-19 has presented unprecedented challenges for public health and disease prevention. The transmission of COVID-19 is very efficient, in which one patient could reportedly transmit the disease to 2 to 3.5 other patients at the early stage [10]11.

At the present time we control the epidemic is associated to the potentiality to generate meaningful data, that in turn will enable us to utilize identical data for mathematical modeling purposes, which are the ideal structure in agreement with forthcoming scenarios [12. Many attempts have been taken in this field to understand the dynamics of epidemiological cycle of the epidemic 10, 13, 14, 15. The rearrangement of the system parameters in a viable way, through the application of limits on the system in obtaining the optimization of a given function, can be executed through the optimal control theory.

Different types of mathematical models have been developed and observed to study the transmission dynamics of the novel coronavirus epidemic with their dynamical behaviors [16, 17, 18, 19, 20, 21, 22, 23, 24, 25, 26, 27,. Data-driven model for the COVID-19 transmission dynamics with the incorporation of distributed time delay has been investigated by Liu et al. 28. Khyar et al. proposed a multi-strain SEIR compartmental model to investigate the interactive dynamics of the novel coronavirus outbreak and showed that their model produce global dynamics 29. The theory of optimal control strategies has been utilized to investigate the COVID-19 disease transmission and the authors showed that optimal doses are needed to curtail the spread of the novel coronavirus diseases [30,31,32. Different types of qualitative behavior has been investigated in different mathematical models $[33,34,35$. Data-driven modeling approach is a powerful tool to investigate the dynamics of the infectious diseases [36.

The use of optimal control theory in infectious disease is very common: while mathematical modeling of the infectious diseases has expressed that amalgamations of hospitalization, isolation and vaccination are usually essential in order to obliterate infectious diseases, the theory of optimal control tells us how they should be managed, by giving the proper times for intervention with proper dosages [37. This optimization technique has also been utilized in some research works within the span of novel coronavirus. The optimal control theory of a modified Susceptible-Exposed-Infected-Recovered (SEIR) mathematical model has been investigated with the goal to aim to study the effectiveness of two inflexible lockdown techniques on the United Kingdom population [38. Another novel coronavirus case studies incorporate the utilization of the theory optimal control in the United States of America [39]. An optimal use of an hypothetical vaccine for novel coronavirus has been also studied [40]. Non-pharmaceutical intervention strategies can be used as a key parameter to maintain the active number of infected populations. 
Here we are fascinated in applying the theory of optimal control has a method to better understand ways to control the progression/development of the novel coronavirus in a case study of the Republic of India by designing optimal disease intervention techniques. Furthermore, we consider some significant questions that are not completely studied in the existing literature. Our model demonstrates the implementation of optimal control technique, to minimize the symptomatic infected class and hospitalized individuals where the response applicability of public health facilities is perpetuated. As the COVID-19 epidemic has exhibited that the public health facility is not only a clinical problem, but also influences the country as a whole 32]. The qualitative behavior of the interactive dynamics of the novel coronavirus epidemic has also been investigated in this manuscript.

The organization of the article is as follows: in the next section, we describe the proposed SAIQJR model. In the same section, we describe the qualitative behavior of the proposed model incorporating positivity, boundedness, computation of basic reproduction number, normalized forward sensitivity analysis for $R_{0}$, existence and stability of the biologically feasible equilibrium points. We also performed the global asymptotic stability of the disease-free equilibrium points. Moreover, we implement the optimal control strategy with three different controls and solve the control problem analytically in the Section 3. In the section 4 , we performed extensive numerical simulations without optimal control and with the implementation of optimal control to validate our theoretical analysis. The paper ends with a through discussion from the view point of novel coronavirus pandemic.

\section{The mathematical model}

We investigate an extended SIR (susceptible-infected-recovered)-type epidemiological model that incorporates the interventions such as quarantine and hospitalization. The epidemiological model stratify the dynamics of six individuals, namely susceptible $(S(t))$, asymptomatic infected $(A(t))$, clinically ill and symptomatic infected $(I(t))$, quarantined $(Q(t))$, isolation or hospitalization $(J(t))$ and the recovered $(R(t))$. Thus, the total population $N(t)$ is given by $N(t)=S(t)+A(t)+I(t)+Q(t)+J(t)+R(t)$. In our model, quarantine represents the separation of SARS-CoV-2 infected individuals from the susceptible population before the progression of the clinical symptoms, while hospitalization delineates the separation of COVID-19 infected populations with such clinical symptoms. A transfer diagram of the proposed novel coronavirus model based on the present situation of India is depicted in the following Figure 1. The transmission dynamics of SARS-CoV-2 is modeled by the following system of coupled non-linear ordinary differential equations:

$$
\frac{d S}{d t}=\Lambda_{s}-\beta_{s}\left(\alpha_{a} A+\alpha_{i} I+\alpha_{j} J\right) S+\beta_{q} Q-\delta S
$$




$$
\begin{aligned}
\frac{d A}{d t} & =\beta_{s}\left(\alpha_{a} A+\alpha_{i} I+\alpha_{j} J\right) S-\sigma_{a} A-\gamma_{a} A-\delta A, \\
\frac{d I}{d t} & =\rho_{i} \sigma_{a} A-\gamma_{i} I-\beta_{i} I-\delta I, \\
\frac{d Q}{d t} & =\left(1-\rho_{i}\right) \sigma_{a} A-\alpha_{q} Q-\beta_{q} Q-\delta Q, \\
\frac{d J}{d t} & =\beta_{i} I+\alpha_{q} Q-\gamma_{j} J-\delta J, \\
\frac{d R}{d t} & =\gamma_{a} A+\gamma_{i} I+\gamma_{j} J-\delta R,
\end{aligned}
$$

subject to non-negative initial conditions

$$
\begin{aligned}
& S(0)=S^{0} \geq 0, \quad A(0)=A^{0} \geq 0, \quad I(0)=I^{0} \geq 0 \\
& Q(0)=Q^{0} \geq 0, \quad J(0)=J^{0} \geq 0, \quad R(0)=R^{0} \geq 0
\end{aligned}
$$

In our mode, we includes the demographic impacts by supposing a proportional natural death rate $\delta>0$ in each of the six individuals of the model. Furthermore, we includes the net inflow of susceptible population at the rate $\Lambda_{s}$ per unit time. This parameter indicates the new births and immigration of the individuals. The susceptible individuals become asymptomatic infected individuals, due to effective contact with exposed class at a rate $\beta_{s}$. The transmission coefficients for the asymptomatic infected, symptomatic infected and hospitalized or isolated infected populations are $\alpha_{a}, \alpha_{i}$, and $\alpha_{j}$ respectively. Among the quarantined individuals, $\alpha_{q} Q$ portions move to the isolation or hospitalization due to seriousness of the diseases, and the $\beta_{q} Q$ part become susceptible to the novel coronavirus diseases after the quarantined period. Here, $\sigma_{a}$ represents the rate at which asymptomatic infected individuals become clinically ill or infectious, $\rho_{i}$ is the rate at which asymptomatic infected individuals become symptomatic infected individuals by a proportion of $\rho_{i}$, and another portion $\left(1-\rho_{i}\right)$ move to the quarantined individuals. The term $\delta$ delineates the natural decay rate of all the individuals, whereas $\beta_{i}$, and $\alpha_{q}$ denotes the symptomatically infected individuals move to the isolated class, and quarantined individuals also move to the isolated class, respectively. The terms $\gamma_{a}, \gamma_{i}$, and $\gamma_{j}$ describes the rates at which asymptomatic infected individuals, clinically ill or symptomatic infected populations, and isolated infected individuals move to the recovered stages, respectively. Our epidemiological model is a system of coupled nonlinear ordinary differential equations. In exercise, some of the parameters may alter in time as control measures are executed or altered. The analytical calculations are studied for the model with constant parameters and all the parameters are assumed to be positive.

\subsection{Positivity and boundedness}

The system of equations (1) is related to novel coronavirus, thus we must have to check whether the state variables are non-negative for all time $t$ with given 


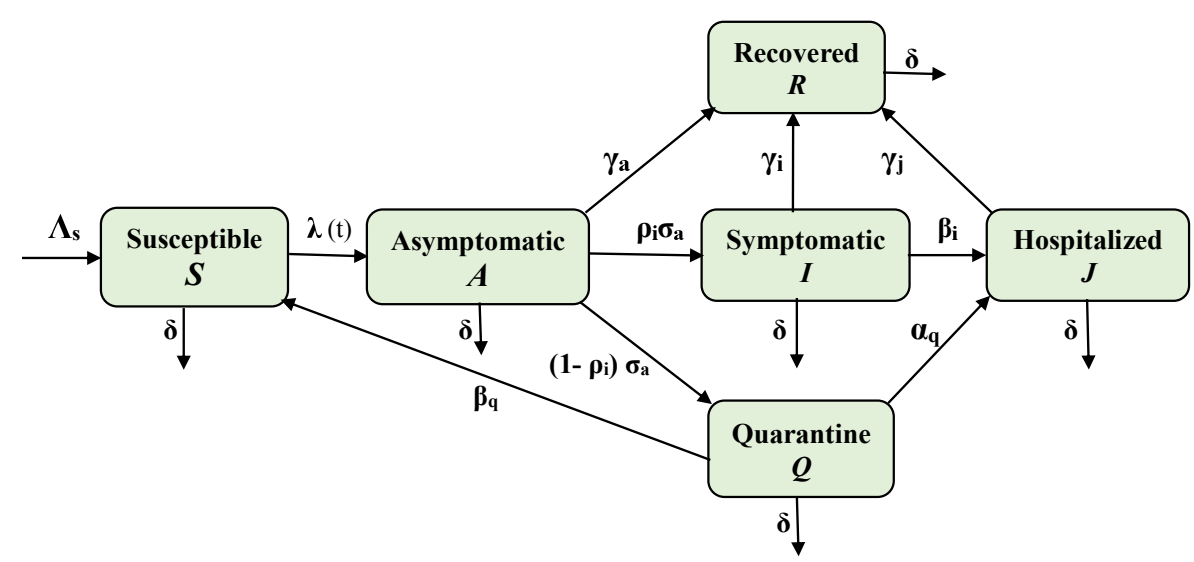

Fig. 1 Schematic diagram of the novel coronavirus model 1 . The epidemiological model includes six sub-populations: susceptible $S$, asymptomatic infected $A$, symptomatic infected $I$, quarantined $Q$, isolated or hospitalized $J$ and recovered $R$ individuals.

initial values (2). Next we shall investigate the solutions of the system (1) are uniformly bounded. In this purpose, we study the following theorem.

Theorem 1 All the solutions $(S(t), A(t), I(t), Q(t), J(t), R(t)) \in \mathbf{R}_{+}^{6}$ of the system (1) with initial conditions (2) remain non-negative and are uniformly bounded in the positively invariant region $\Omega$ for all time $t \geq 0$.

Proof Let us consider $(S(t), A(t), I(t), Q(t), J(t), R(t)) \in \mathbf{R}_{+}^{6}$ be a solution of (1) for $t \in\left[0, t_{0}\right)$, where $t_{0}>0$. Now we assume that there exists some time $t_{1}>0$ such that $S\left(t_{1}\right)=0$ and for the first time $t_{1}, d S\left(t_{1}\right) / d t \leq 0$. But this assumption ends with a contradiction, since $d S\left(t_{1}\right) / d t \leq 0=\Lambda_{s}$ for the model system (1). Therefore $S>0$, for all $t \geq 0$.

From the first equation of (1), we obtain

$$
\begin{aligned}
\frac{d S}{d t} & =\Lambda_{s}-\beta_{s}\left[\alpha_{i} I+\alpha_{a} A+\alpha_{j} J\right] S+\beta_{q} Q-\delta S, \\
& \geq \Lambda_{s}-\psi(t) S,
\end{aligned}
$$

where, $\psi(t)=\beta_{s}\left[\alpha_{i} I+\alpha_{a} A+\alpha_{j} J\right]+\delta$. After integration, we get

$S(t)=S^{0} \exp \left(-\int_{0}^{t} \psi(s) d s\right)+\Lambda_{s} \exp \left(-\int_{0}^{t} \psi(s) d s\right) \times \int_{0}^{t} e^{\int_{0}^{s} \psi(u) d u} d s>0$.

Therefore for all $t \in\left[0, t_{0}\right)$, we obtain that $S>0$. The second equation of 11) can be written as

$$
\frac{d A}{d t} \geq-\left(\sigma_{a}+\gamma_{a}+\delta\right) A
$$

this leads to

$$
A(t) \geq A^{0} \exp \left(-\int_{0}^{t}\left(\sigma_{a}+\gamma_{a}+\delta\right) d s\right) \geq 0
$$


Third equation of (1) gives

$$
\frac{d I}{d t} \geq-\left(\gamma_{i}+\beta_{i}+\delta\right) I
$$

which implies that

$$
I(t) \geq I^{0} \exp \left(-\int_{0}^{t}\left(\gamma_{i}+\beta_{i}+\delta\right) d s\right) \geq 0 .
$$

In the similar manner, we can write the fourth and fifth equations of (1) can be expressed as

$$
\frac{d Q}{d t} \geq-\left(\alpha_{q}+\beta_{q}+\delta\right) Q \text { and } \frac{d J}{d t} \geq-\left(\gamma_{j}+\delta\right) J .
$$

Then after integration, we have

$$
\begin{aligned}
& Q(t) \geq Q^{0} \exp \left(-\int_{0}^{t}\left(\alpha_{q}+\beta_{q}+\delta\right) d s\right) \geq 0, \\
& J(t) \geq J^{0} \exp \left(-\int_{0}^{t}\left(\gamma_{j}+\delta\right) d s\right) \geq 0 .
\end{aligned}
$$

In the same way, the last equation of (1) can be expressed as

$$
\frac{d R}{d t} \geq-\delta R
$$

which gives

$$
R(t) \geq R^{0} \exp \left(-\int_{0}^{t} \delta d s\right) \geq 0 .
$$

Therefore, the solutions $(S, A, I, Q, J, R)$ of (1) satisfying the initial conditions for all $t \in\left[0, t_{0}\right), t_{0}>0$ remain non-negative in the region $\left[0, t_{0}\right)$.

Now, we prove the boundedness of the solutions $(S, A, I, Q, J, R)$ of (1). The positivity of the solutions implies that

$$
\frac{d S}{d t} \leq \Lambda_{s}-\delta S .
$$

From the above equation, we can write that

$$
\limsup _{t \rightarrow \infty} S \leq \frac{\Lambda_{s}}{\delta},
$$

and consequently

$$
S \leq \frac{\Lambda_{s}}{\delta}
$$


Here, we consider the total population $N$ as $N=S+A+I+Q+J+R$.

Taking derivative of (1), we have

this leads to

$$
\frac{d N}{d t} \leq \Lambda_{s}-\delta N
$$

$$
\limsup _{t \rightarrow \infty} N \leq \frac{\Lambda_{s}}{\delta}
$$

Thus, we obtain $N \leq \Lambda_{s} / \delta$ and consequently $S+A+I+Q+J+R \leq \Lambda_{s} / \delta$. Therefore all the solution trajectories $(S, A, I, Q, J, R)$ satisfying the initial conditions are uniformly bounded in $\mathbf{R}_{+}^{6}$ and in the region

$$
\Omega:=\left\{(S, A, I, Q, J, R) \in \mathbf{R}_{+}^{6}: S \leq \frac{\Lambda_{s}}{\delta}, S+A+I+Q+J+R \leq \frac{\Lambda_{s}}{\delta}\right\}(3)
$$

Therefore all the solutions of 11 initiating in $\mathbf{R}_{+}^{6}$ will enter into the region $\Omega$ in a finite time frame.

\subsection{Basic reproduction number}

The system of equations (1) has a unique disease-free equilibrium (DFE) point $E_{0}$ where $A=0, I=0, Q=0$ and $J=0$, that is, $E_{0}$ is defined by $E_{0}\left(S^{0}, 0,0,0,0, R^{0}\right)$ where $S^{0}=\Lambda_{s} / \delta$ and $R^{0}=0$. Now we determine the basic reproduction number $R_{0}$ of our compartmental model (1) using the next generation matrix and the approach developed by Diekmann et al. [41] and van den Driessche \& Watmough [42. To do this, we consider the matrix $\mathcal{F}$ and a non-singular $M$ matrix $\mathcal{V}$, represents the creation of new-infection and transition part respectively, for the equations (1). We linearized infectious subsystem at the DFE $E_{0}\left(S^{0}, 0,0,0,0,0\right)$ as $\dot{x}=\mathcal{F}-\mathcal{V}$, where $x=(A, I, Q, J)$ are described by $\mathcal{F}, \mathcal{V}$ are given by

$$
\mathcal{F}=\left(\begin{array}{c}
\beta_{s} S\left[\alpha_{a} A+\alpha_{i} I+\alpha_{j} J\right] \\
0 \\
0 \\
0
\end{array}\right) \text { and } \mathcal{V}=\left(\begin{array}{c}
\left(\sigma_{a}+\gamma_{a}+\delta\right) A \\
-\rho_{i} \sigma_{a} A+\left(\gamma_{i}+\beta_{i}+\delta\right) I \\
-\left(1-\rho_{i}\right) \sigma_{a} A+\left(\alpha_{q}+\beta_{q}+\delta\right) Q \\
-\beta_{i} I-\alpha_{q} Q+\left(\gamma_{j}+\delta\right) J
\end{array}\right)
$$

The Jacobian matrix of the model (1) can be calculated at the disease-free steady state $(A=I=Q=J=0)$, we get

$$
\begin{aligned}
F & =\left(\begin{array}{cccc}
\beta_{s} \alpha_{a} S^{0} & \beta_{s} \alpha_{i} S^{0} & 0 & \beta_{s} \alpha_{j} S^{0} \\
0 & 0 & 0 & 0 \\
0 & 0 & 0 & 0 \\
0 & 0 & 0 & 0
\end{array}\right) \text { and } \\
V & =\left(\begin{array}{cccc}
\sigma_{a}+\gamma_{a}+\delta & 0 & 0 & 0 \\
-\rho_{i} \sigma_{a} & \gamma_{i}+\beta_{i}+\delta & 0 & 0 \\
-\left(1-\rho_{i}\right) \sigma_{a} & 0 & \alpha_{q}+\beta_{q}+\delta & 0 \\
0 & -\beta_{i} & -\alpha_{q} & \gamma_{j}+\delta
\end{array}\right) .
\end{aligned}
$$


Thus we can derive the next generation matrix $\rho\left(F V^{-1}\right)$ and the basic reproduction number $R_{0}$ is the spectral radius of the next generation matrix, we have

$$
\begin{aligned}
R_{0}=\rho\left(F V^{-1}\right) & =\frac{\beta_{s} \alpha_{a} S^{0}}{\left(\sigma_{a}+\gamma_{a}+\delta\right)}+\frac{\beta_{s} \alpha_{i} \rho_{i} \sigma_{a} S^{0}}{\left(\sigma_{a}+\gamma_{a}+\delta\right)\left(\gamma_{i}+\beta_{i}+\delta\right)} \\
& +\frac{\beta_{s} \beta_{i} \alpha_{j} \rho_{i} \sigma_{a} S^{0}}{\left(\sigma_{a}+\gamma_{a}+\delta\right)\left(\gamma_{i}+\beta_{i}+\delta\right)\left(\gamma_{j}+\delta\right)} \\
& +\frac{\beta_{s} \alpha_{j} \alpha_{q}\left(1-\rho_{i}\right) \sigma_{a} S^{0}}{\left(\sigma_{a}+\gamma_{a}+\delta\right)\left(\alpha_{q}+\beta_{q}+\delta\right)\left(\gamma_{j}+\delta\right)}
\end{aligned}
$$

The basic reproduction number $\left(R_{0}\right)$ is an epidemiologic measurement and it help us to convey predictions about transmissibility of emergent infectious disease like COVID-19.

\subsection{Sensitivity analysis of $R_{0}$}

In this subsection, we study the sensitivity of the system parameters associated to the basic reproduction number $R_{0}$ to analyze their robustness corresponding to the $S A I Q J R$ model system prediction as the initial transmission of any epidemic is associated to the reproduction number $R_{0}$. Sensitivity analysis aids to recognize the most effective parameters with reference to $R_{0}$. Since a little perturbation to the value of the most sensitive parameters can impose a great effect in any epidemiological system. Thus, to convey predictions about the prevalence of the diseases, persistence and reduction of the transmission of COVID-19 infection we performed the normalized forward sensitivity analysis.

Definition 1 The normalized forward sensitivity index of any model parameter, $m$ related with $R_{0}$ differentially is defined as:

$$
\Upsilon_{m}^{R_{0}}:=\frac{\partial m}{\partial R_{0}} \times \frac{R_{0}}{m} .
$$

The normalized forward sensitivity index may be dependent on the model parameters or may be independent (constant value).

Now, we calculate the normalized forward sensitivity indices of the system parameters associated with $R_{0}$ and the indices are listed in Table 1 .

From the Table 1 it can be noticed that some of the sensitivity indices are positive and the rest are of negative. The positive or negative sign represents that the perturbation to these parameters can increase or decrease the value of $R_{0}$, respectively. As for example, it can be observed that the normalized forward sensitivity index of $\alpha_{a}$ is $\Upsilon_{\alpha_{a}}^{R_{0}}=+0.6768$. This sensitivity index $\Upsilon_{\alpha_{a}}^{R_{0}}$ indicates that if the value of $\alpha_{a}$ would be increased by $10 \%$, as a result the value of $R_{0}$ would be increased by $6.768 \%$. Again it is obtained that the sensitivity index of $\delta$ is given by $\Upsilon_{\delta}^{R_{0}}=-0.92141$. The index $\Upsilon_{\delta}^{R_{0}}$ is indicating 
Table 1 Sensitivity indices of the $S A I Q J R$ model parameters associated with $R_{0}$.

\begin{tabular}{|c|c|c|c|c|c|c|c|}
\hline Parameter & $\Lambda_{s}$ & $\beta_{s}$ & $\alpha_{a}$ & $\alpha_{j}$ & $\alpha_{q}$ & $\rho_{i}$ & $\beta_{i}$ \\
\hline $\begin{array}{c}\text { Sensitivity } \\
\text { Index }\end{array}$ & 1.00000 & 1.00000 & 0.67680 & 0.32315 & 0.31271 & 0.00482 & 0.00029 \\
\hline \hline Parameter & $\alpha_{i}$ & $\gamma_{i}$ & $\beta_{q}$ & $\sigma_{a}$ & $\gamma_{a}$ & $\gamma_{j}$ & $\delta$ \\
\hline $\begin{array}{c}\text { Sensitivity } \\
\text { Index }\end{array}$ & 0.00005 & -0.00003 & -0.01188 & -0.22556 & -0.25519 & -0.32154 & -0.92141 \\
\hline
\end{tabular}

the fact that $10 \%$ increase to the value of $\delta$ will draw $9.2141 \%$ decrease to the value of $R_{0}$. From the Table 1 it is found that the normalized forward sensitivity indices of the parameters $\Lambda_{s}, \beta_{s}, \alpha_{a}, \alpha_{i}, \alpha_{j}, \alpha_{q}, \beta_{i}$ and $\rho_{i}$ have positive influence on $R_{0}$ (that is, increase the value of $R_{0}$ ) and the rest of the indices (the parameters $\gamma_{i}, \beta_{q}, \sigma_{a}, \gamma_{a}, \gamma_{j}$ and $\delta$ ) create negative influence on $R_{0}$ (that is, decrease the value of $R_{0}$ ). In this scenario, we notice that the most effective parameters of the system 1 are the disease transmission rate $\left(\beta_{s}\right)$ and the recruitment rate of susceptible individuals $\left(\Lambda_{s}\right)$ which are inducing positive impact on $R_{0}$. Besides, the natural mortality rate of all the individuals $(\delta)$ is also very influencing parameter imposing negative impact on $R_{0}$. We have to pay more attention to these three highly sensitive parameters to predict the COVID-19 disease transmission. We have shown this behavior of the parameters with reference to $R_{0}$ in Fig. 5 .

\subsection{Local stability in terms of the basic reproduction number}

In this subsection we studied the local asymptotic stability of the system (1) of with reference to the state variables are $A, I, Q, J$, that is, the variational matrix related to these variables of the system (1) is follows

$$
J_{B}=\left(\begin{array}{cccc}
\beta_{s} \alpha_{a} S^{0}-\left(\sigma_{a}+\gamma_{a}+\delta\right) & \beta_{s} \alpha_{i} S^{0} & 0 & \beta_{s} \alpha_{j} S^{0} \\
\rho_{i} \sigma_{a} & -\left(\gamma_{i}+\beta_{i}+\delta\right) & 0 & 0 \\
\left(1-\rho_{i}\right) \sigma_{a} & 0 & -\left(\alpha_{q}+\beta_{q}+\delta\right) & 0 \\
0 & \beta_{i} & \alpha_{q} & -\left(\gamma_{j}+\delta\right)
\end{array}\right)
$$

From the above Jacobian matrix $J_{B}$, we assume that $\varpi$ be the eigenvalue of the matrix. Thus, the eigenvalues of the Jacobian matrix $J_{B}$ are the roots of the following characteristic equation

$$
P(\varpi)=\varpi^{4}+\zeta_{1} \varpi^{3}+\zeta_{2} \varpi^{2}+\zeta_{3} \varpi+\zeta_{4},
$$

where

$$
\begin{aligned}
\zeta_{1}= & \varrho_{1}+\varrho_{2}+\varrho_{3}+\varrho_{4}-\beta_{s} \alpha_{a} S^{0}, \\
\zeta_{2}= & \varrho_{1}\left(\varrho_{2}+\varrho_{3}+\varrho_{4}\right)+\varrho_{2} \varrho_{3}+\varrho_{2} \varrho_{3}+\varrho_{3} \varrho_{4}-\left(\varrho_{2}+\varrho_{3}+\varrho_{4}\right) \beta_{s} \alpha_{a} S^{0}-\beta_{s} \rho_{i} \sigma_{a} \alpha_{i} S^{0}, \\
\zeta_{3}= & \varrho_{1}\left(\varrho_{2} \varrho_{3}+\varrho_{2} \varrho_{3}+\varrho_{3} \varrho_{4}\right)+\varrho_{2} \varrho_{3} \varrho_{4}+\left(\varrho_{2} \varrho_{3}+\varrho_{2} \varrho_{3}+\varrho_{3} \varrho_{4}\right) \beta_{s} \alpha_{a} S^{0} \\
& -\left(\varrho_{3}+\varrho_{4}\right) \beta_{s} \rho_{i} \sigma_{a} \alpha_{i} S^{0}-\beta_{s} \rho_{i} \sigma_{a} \alpha_{j} \beta_{i} S^{0}-\beta_{s} \alpha_{q} \alpha_{j} \sigma_{a}\left(1-\rho_{i}\right) S^{0}, \\
\zeta_{4}= & \varrho_{1} \varrho_{2} \varrho_{3} \varrho_{4}-\varrho_{2} \varrho_{3} \varrho_{4} \beta_{s} \alpha_{a} S^{0}-\varrho_{3} \beta_{s} \rho_{i} \sigma_{a} \alpha_{j} \beta_{i} S^{0}-\varrho_{2} \beta_{s} \alpha_{q} \alpha_{j} \sigma_{a}\left(1-\rho_{i}\right) S^{0}-\varrho_{3} \varrho_{4} \beta_{s} \rho_{i} \sigma_{a} \alpha_{i} S^{0},
\end{aligned}
$$


with $\varrho_{1}=\sigma_{a}+\gamma_{a}+\delta, \quad \varrho_{2}=\gamma_{i}+\beta_{i}+\delta, \quad \varrho_{3}=\alpha_{q}+\beta_{q}+\delta, \quad \varrho_{4}=\gamma_{j}+\delta$. Due to the well-known Lienard-Chipard test [43,44, all the roots are negative or have negative real part, provided that, the following conditions are hold:

a) $\zeta_{\iota}>0, \quad \iota=1,2,3,4$

b) $\zeta_{1} \zeta_{2}>\zeta_{3}$

To verify these expressions of the Lienard-Chipard test, we express the coefficients $\zeta_{1}, \zeta_{2}, \zeta_{3}$, and $\zeta_{4}$ of the above characteristic equation (6) in terms $R_{0}$ is written as

$$
\begin{aligned}
\zeta_{1}= & \varrho_{1}\left(1-R_{0}\right)+\varrho_{2}+\varrho_{3}+\varrho_{4}+\frac{\beta_{s} \sigma_{a} S^{0}}{\varrho_{2}}\left[\alpha_{i} \rho_{i}+\frac{\alpha_{j} \rho_{i} \beta_{i}}{\varrho_{4}}+\frac{\varrho_{2} \alpha_{j} \alpha_{q}\left(1-\rho_{i}\right)}{\varrho_{3} \varrho_{4}}\right], \\
\zeta_{2}= & \varrho_{1}\left(\varrho_{2}+\varrho_{3}+\varrho_{4}\right)\left(1-R_{0}\right)+\varrho_{2} \varrho_{3}+\varrho_{2} \varrho_{3}+\varrho_{3} \varrho_{4}+\beta_{s} \rho_{i} \sigma_{a} \alpha_{j} \beta_{i} S^{0}\left[\frac{1}{\varrho_{2}}+\frac{1}{\varrho_{4}}+\frac{\varrho_{3}}{\varrho_{2} \varrho_{4}}\right] \\
& +\beta_{s} \alpha_{q} \alpha_{j} \sigma_{a}\left(1-\rho_{i}\right) S^{0}\left[\frac{1}{\varrho_{3}}+\frac{1}{\varrho_{4}}+\frac{\varrho_{2}}{\varrho_{3} \varrho_{4}}\right]+\beta_{s} \rho_{i} \sigma_{a} \alpha_{i} S^{0}\left[\frac{\varrho_{3}}{\varrho_{2}}+\frac{\varrho_{4}}{\varrho_{2}}\right], \\
\zeta_{3}= & \varrho_{1}\left(\varrho_{2} \varrho_{3}+\varrho_{2} \varrho_{3}+\varrho_{3} \varrho_{4}\right)\left(1-R_{0}\right)+\varrho_{2} \varrho_{3} \varrho_{4}+\varrho_{3} \beta_{s} \rho_{i} \sigma_{a} \alpha_{j} \beta_{i} S^{0}\left[\frac{1}{\varrho_{2}}+\frac{1}{\varrho_{4}}\right] \\
& +\varrho_{2} \beta_{s} \alpha_{q} \alpha_{j} \sigma_{a}\left(1-\rho_{i}\right) S_{0}\left[\frac{1}{\varrho_{3}}+\frac{1}{\varrho_{4}}\right]+\frac{\varrho_{3} \varrho_{4}}{\varrho_{2}} \beta_{s} \rho_{i} \sigma_{a} \alpha_{i} S^{0}, \\
\zeta_{4}= & \varrho_{1} \varrho_{2} \varrho_{3} \varrho_{4}\left(1-R_{0}\right) .
\end{aligned}
$$

Furthermore, we also calculate the expression $\zeta_{1} \zeta_{2}-\zeta_{3}$ in terms of $R_{0}$ as follows:

$$
\begin{aligned}
& \zeta_{1} \zeta_{2}-\zeta_{3}=\varrho_{1}\left(\varrho_{2}+\varrho_{3}+\varrho_{4}\right)^{2}\left(1-R_{0}\right)+\varrho_{1}^{2}\left(\varrho_{2}+\varrho_{3}+\varrho_{4}\right)\left(1-R_{0}\right)^{2}+\left(\varrho_{3}+\varrho_{4}\right)\left(\varrho_{2} \varrho_{3}+\varrho_{2}^{2}+\varrho_{2} \varrho_{4}\right. \\
& \left.+\varrho_{3} \varrho_{4}\right)+\left(\varrho_{1}+\frac{\varrho_{1} \varrho_{3}}{\varrho_{2}}+\frac{\varrho_{1} \varrho_{4}}{\varrho_{2}}\right)\left(1-R_{0}\right) \beta_{s} \sigma_{a} S^{0}\left[\alpha_{i} \rho_{i}+\frac{\alpha_{j} \rho_{i} \beta_{i}}{\varrho_{4}}+\frac{\varrho_{2} \alpha_{j} \alpha_{q}\left(1-\rho_{i}\right)}{\varrho_{3} \varrho_{4}}\right] \\
& +\left(\frac{\varrho_{2}}{\varrho_{4}}+\frac{\varrho_{4}}{\varrho_{2}}+\frac{\varrho_{3}}{\varrho_{4}}+\frac{\varrho_{3}}{\varrho_{2}}+\frac{\varrho_{3}^{2}}{\varrho_{2} \varrho_{4}}\right) \beta_{s} \rho_{i} \sigma_{a} \alpha_{j} \beta_{i} S^{0}+\left(\frac{\varrho_{1} \varrho_{3}}{\varrho_{2}}+\frac{\varrho_{1} \varrho_{4}}{\varrho_{2}}\right)\left(1-R_{0}\right) \beta_{s} \rho_{i} \sigma_{a} \alpha_{i} S^{0} \\
& +\frac{1}{\varrho_{2}}\left(\frac{1}{\varrho_{2}}+\frac{1}{\varrho_{4}}+\frac{\varrho_{3}}{\varrho_{2} \varrho_{4}}\right) \beta_{s}^{2} \rho_{i} \sigma_{a}^{2} \alpha_{j} \beta_{i} S^{0^{2}}\left[\alpha_{i} \rho_{i}+\frac{\alpha_{j} \rho_{i} \beta_{i}}{\varrho_{4}}+\frac{\varrho_{2} \alpha_{j} \alpha_{q}\left(1-\rho_{i}\right)}{\varrho_{3} \varrho_{4}}\right] \\
& +\frac{1}{\varrho_{2}}\left(\frac{1}{\varrho_{3}}+\frac{1}{\varrho_{4}}+\frac{\varrho_{2}}{\varrho_{3} \varrho_{4}}\right) \beta_{s}^{2}\left(1-\rho_{i}\right) \sigma_{a}^{2} \alpha_{q} \alpha_{j} S^{0^{2}}\left[\alpha_{i} \rho_{i}+\frac{\alpha_{j} \rho_{i} \beta_{i}}{\varrho_{4}}+\frac{\varrho_{2} \alpha_{j} \alpha_{q}\left(1-\rho_{i}\right)}{\varrho_{3} \varrho_{4}}\right] \\
& +\left(\frac{\varrho_{3}}{\varrho_{2}^{2}}+\frac{\varrho_{4}}{\varrho_{2}^{2}}\right) \beta_{s}^{2} \rho_{i} \sigma_{a}^{2} \alpha_{i} S^{0^{2}}\left[\alpha_{i} \rho_{i}+\frac{\alpha_{j} \rho_{i} \beta_{i}}{\varrho_{4}}+\frac{\varrho_{2} \alpha_{j} \alpha_{q}\left(1-\rho_{i}\right)}{\varrho_{3} \varrho_{4}}\right] \\
& +\left(\frac{\varrho_{1}}{\varrho_{2}}+\frac{\varrho_{1}}{\varrho_{4}}+\frac{\varrho_{1} \varrho_{3}}{\varrho_{2} \varrho_{4}}\right)\left(1-R_{0}\right) \beta_{s} \rho_{i} \sigma_{a} \alpha_{j} \beta_{i} S^{0}+\frac{\varrho_{3} \varrho_{4}}{\varrho_{2}} \beta_{s} \sigma_{a} S^{0}\left[\frac{\alpha_{j} \rho_{i} \beta_{i}}{\varrho_{4}}+\frac{\varrho_{2} \alpha_{j} \alpha_{q}\left(1-\rho_{i}\right)}{\varrho_{3} \varrho_{4}}\right] \\
& +\left(\frac{\varrho_{1}}{\varrho_{3}}+\frac{\varrho_{1}}{\varrho_{4}}+\frac{\varrho_{1} \varrho_{2}}{\varrho_{3} \varrho_{4}}\right)\left(1-R_{0}\right) \beta_{s}\left(1-\rho_{i}\right) \sigma_{a} \alpha_{q} \alpha_{j} S^{0}+\left(\varrho_{2}+\varrho_{3}+\varrho_{4}\right) \frac{\varrho_{3}+\varrho_{4}}{\varrho_{2}} \beta_{s} \alpha_{i} \rho_{i} \sigma_{a} S^{0} \\
& +\left(\varrho_{3}+\varrho_{4}\right)\left(\frac{1}{\varrho_{3}}+\frac{1}{\varrho_{4}}+\frac{\varrho_{2}}{\varrho_{3} \varrho_{4}}\right) \beta_{s}\left(1-\rho_{i}\right) \sigma_{a} \alpha_{q} \alpha_{j} S^{0}+2 \beta_{s} \rho_{i} \sigma_{a} \alpha_{j} \beta_{i} S^{0} \\
& +\left(\varrho_{3}+\varrho_{4}\right) \beta_{s} \sigma_{a} S^{0}\left[\alpha_{i} \rho_{i}+\frac{\alpha_{j} \rho_{i} \beta_{i}}{\varrho_{4}}+\frac{\varrho_{2} \alpha_{j} \alpha_{q}\left(1-\rho_{i}\right)}{\varrho_{3} \varrho_{4}}\right] .
\end{aligned}
$$


From the above expression, we can conclude that if $R_{0}<1$, then the LienardChipard test holds successfully and thus the disease-free steady state $E_{0}\left(S^{0}, 0\right.$, $0,0,0,0)$ is stable. For $R_{0}>1$, we obtain $\zeta_{4}<0$ due to Descartes' rule of signs, we decide that at least one of eigenvalues is non-negative. Hence, the model equations (1) is unstable. The result can be summarized in the following theorem.

Theorem 2 The disease-free steady state $E_{0}\left(S^{0}, 0,0,0,0,0\right)$ of (1) is locally asymptotically stable for $R_{0}<1$ and unstable for $R_{0}>1$.

2.5 Existence of endemic steady state

In this subsection, we determine the endemic steady state $E^{*}\left(S^{*}, A^{*}, I^{*}, Q^{*}, J^{*}, R^{*}\right)$ with their feasibility conditions, where

$$
\begin{aligned}
S^{*} & =\frac{1}{R_{0}}, I^{*}=\frac{\sigma_{a} \rho_{i}}{\left(\gamma_{i}+\beta_{i}+\delta\right)} A^{*}, Q^{*}=\frac{\sigma_{a}\left(1-\rho_{i}\right)}{\left(\alpha_{q}+\beta_{q}+\delta\right)} A^{*}, \\
J^{*} & =\left[\frac{\beta_{i} \sigma_{a} \rho_{i}}{\left(\gamma_{i}+\beta_{i}+\delta\right)\left(\gamma_{j}+\delta\right)}+\frac{\alpha_{q} \sigma_{a}\left(1-\rho_{i}\right)}{\left(\alpha_{q}+\beta_{q}+\delta\right)\left(\gamma_{j}+\delta\right)}\right] A^{*} \\
R^{*} & =\frac{1}{\delta}\left[\gamma_{a}+\frac{\sigma_{a} \rho_{i}}{\left(\gamma_{i}+\beta_{i}+\delta\right)}+\frac{\beta_{i} \sigma_{a} \rho_{i}}{\left(\gamma_{i}+\beta_{i}+\delta\right)\left(\gamma_{j}+\delta\right)}+\frac{\alpha_{q} \sigma_{a}\left(1-\rho_{i}\right)}{\left(\alpha_{q}+\beta_{q}+\delta\right)\left(\gamma_{j}+\delta\right)}\right] A^{*}, \\
A^{*} & =\frac{\left(\alpha_{q}+\beta_{q}+\delta\right)\left[\left(\Lambda_{s}-\delta\right)+\delta\left(1-\frac{1}{R_{0}}\right)\right]}{\left(\sigma_{a}+\gamma_{a}+\delta\right)\left(\alpha_{q}+\beta_{q}+\delta\right)-\beta_{q}\left(1-\rho_{i}\right) \sigma_{a}} .
\end{aligned}
$$

The system (1) has a unique biologically feasible interior equilibrium point $E^{*}$, if the following conditions hold:
i) $R_{0}>1$,
ii) $S^{0}>1$,
iii) $\left(\sigma_{a}+\gamma_{a}+\delta\right)\left(\alpha_{q}+\beta_{q}+\delta\right)>\beta_{q}\left(1-\rho_{i}\right) \sigma_{a}$.

2.6 Global stability analysis of the disease-free steady state

Theorem 3 The disease-free steady state $E_{0}\left(\frac{\Lambda_{s}}{\delta}, 0,0,0,0,0\right)$ of 11 is globally asymptotic stable if $R_{0}<1$ and unstable if $R_{0}>1$.

Proof From the system of equations (1) it can be observed that the diseasefree compartments of (1) are $S, R$ and the infected compartments are $A, I, Q$ and $J$. We can arrange the system equations (1) as follows:

$$
\begin{aligned}
& \frac{d X}{d t}=P(X, Y), \\
& \frac{d Y}{d t}=G(X, Y), \text { and } G(X, 0)=0,
\end{aligned}
$$

where

$$
X=(S, R) \in \mathbf{R}_{+}^{2}, \quad Y=(A, I, Q, J) \in \mathbf{R}_{+}^{4} .
$$


Following the method developed by Castillo-Chavez [45], we shall try to investigate the global stability of the disease-free steady state $E_{0}\left(\Lambda_{s} / \delta, 0,0,0,0,0\right)$ and to compute this, the system equations (1) must have to satisfy the following two conditions:

1. $\frac{d X}{d t}=P(X, 0), X^{*}$ is globally asymptotically stable.

2. $G(X, Y)=K Y-\widehat{G}(X, Y), \widehat{G}(X, Y) \geq 0$, where $K=D_{Y} G\left(X^{*}, 0\right)$ is the Metzler Matrix and $(X, Y) \in \Omega$, the biologically feasible and attracting region where all the solutions trajectories will enter into the interior of this region and will never leave it.

Therefore the system (1) can be rewritten as

$$
\begin{aligned}
& P(X, 0)=\left(\begin{array}{c}
\Lambda_{s}-\delta S \\
0
\end{array}\right), \\
& K=\left(\begin{array}{cccc}
-\left(\sigma_{a}+\gamma_{a}+\delta\right)+\beta_{s} \alpha_{a} & \beta_{s} \alpha_{i} & 0 & \beta_{s} \alpha_{j} \\
\rho_{i} \sigma_{a} & -\left(\gamma_{i}+\beta_{i}+\delta\right) & 0 & 0 \\
\left(1-\rho_{i}\right) \sigma_{a} & 0 & -\left(\alpha_{q}+\beta_{q}+\delta\right) & 0 \\
0 & \beta_{i} & \alpha_{q} & -\left(\gamma_{j}+\delta_{j}\right)
\end{array}\right) \\
& \text { and } \widehat{G}=\left(\begin{array}{c}
\beta_{s} \alpha_{a} A(1-S)+\beta_{s} \alpha_{i} I(1-S)+\beta_{s} \alpha_{j} J(1-S) \\
0 \\
0 \\
0
\end{array}\right)
\end{aligned}
$$

Therefore it can be observed that for $(X, Y) \in \Omega, \widehat{G}(X, Y) \geq 0$ and also we can find that $X^{*}=\left(\Lambda_{s} / \delta, 0\right)$ is globally asymptotic stable steady state of the limiting system, $\frac{d X}{d t}=P(X, 0)$. Accordingly, the two conditions are satisfied and in this way the disease-free steady state $E_{0}\left(\Lambda_{s} / \delta, 0,0,0,0,0\right)$ is globally asymptotic stable, when $R_{0}<1$.

2.7 Local stability of endemic steady state

Theorem 4 The unique interior steady state $E^{*}\left(S^{*}, A^{*}, I^{*}, Q^{*}, J^{*}, R^{*}\right)$ is locally asymptotically stable if $R_{0}>1$.

The standard linearization technique of the $S A I Q J R$ model around the unique interior equilibrium point is very laborious mathematically. Thus, we use the center manifold theory [45] to investigate the local asymptotic stability of the interior infection steady state $E^{*}$. To apply this method, we use the following simplification and change of variables are made at first. Let $S=\chi_{1}, A=\chi_{2}$, 
$I=\chi_{3}, Q=\chi_{4}, J=\chi_{5}, R=\chi_{6}$, using this following vector notation, the system (1) can be written in this following form as

$$
\begin{aligned}
\frac{d \chi_{1}}{d t} & =\Lambda_{s}-\beta_{s}\left(\alpha_{a} \chi_{2}+\alpha_{i} \chi_{3}+\alpha_{j} \chi_{5}\right) \chi_{1}+\beta_{q} \chi_{4}-\delta \chi_{1} \equiv \Theta_{1} \\
\frac{d \chi_{2}}{d t} & =\beta_{s}\left(\alpha_{a} \chi_{2}+\alpha_{i} \chi_{3}+\alpha_{j} \chi_{5}\right) \chi_{1}-\left(\sigma_{a}+\gamma_{a}+\delta\right) \chi_{2} \equiv \Theta_{2}, \\
\frac{d \chi_{3}}{d t} & =\rho_{i} \sigma_{a} \chi_{2}-\left(\gamma_{i}+\beta_{i}+\delta\right) \chi_{3} \equiv \Theta_{3} \\
\frac{d \chi_{4}}{d t} & =\left(1-\rho_{i}\right) \sigma_{a} \chi_{2}-\left(\alpha_{q}+\beta_{q}+\delta\right) \chi_{4} \equiv \Theta_{4}, \\
\frac{d \chi_{5}}{d t} & =\beta_{i} \chi_{3}+\alpha_{q} \chi_{4}-\left(\gamma_{j}+\delta\right) \chi_{5} \equiv \Theta_{5}, \\
\frac{d \chi_{6}}{d t} & =\gamma_{a} \chi_{2}+\gamma_{i} \chi_{3}+\gamma_{j} \chi_{5}-\delta \chi_{6} \equiv \Theta_{6},
\end{aligned}
$$

The variational matrix of $(1)$ around the disease-free fixed point $E_{0}\left(S^{0}, 0,0,0,0,0\right)$ is given by

$$
\mathcal{J}_{E_{0}}^{*}=\left(\begin{array}{cccccc}
-\delta & 0 & -\beta_{s} \alpha_{a} S^{0} & -\beta_{q}-\beta_{s} \alpha_{i} S^{0} & 0 \\
0 & -\left(\varrho_{1}-\beta_{s} \alpha_{a} S^{0}\right) & \beta_{s} \alpha_{a} S^{0} & 0 & \beta_{s} \alpha_{i} S^{0} & 0 \\
0 & q_{i} \sigma_{a} & -\varrho_{2} & 0 & 0 & 0 \\
0 & \left(1-q_{i}\right) \sigma_{a} & 0 & -\varrho_{3} & 0 & 0 \\
0 & 0 & \beta_{i} & \alpha_{q} & -\varrho_{4} & 0 \\
0 & \gamma_{a} & \gamma_{i} & 0 & \gamma_{j} & -\delta
\end{array}\right) .
$$

Here we used $\varrho_{1}, \varrho_{2}, \varrho_{3}$, and $\varrho_{4}$ that are already mentioned as earlier before and $S^{0}=\Lambda_{s} / \delta$. Considering $\beta_{s}$ as the bifurcation parameter, we calculate $\beta_{s}$ by using $R_{0}=1$. Now we compute $\beta_{s}$ as $\beta_{s}=\beta_{s}^{*}$ is given by

$$
\beta_{s}^{*}=\frac{S^{0} \varrho_{1} \varrho_{2} \varrho_{3} \varrho_{4}}{\alpha_{a} \varrho_{2} \varrho_{3} \varrho_{4}+\alpha_{i} \rho_{i} \sigma_{a} \varrho_{3} \varrho_{4}+\beta_{i} \alpha_{j} \rho_{i} \sigma_{a} \varrho_{3}+\alpha_{j} \alpha_{q}\left(1-\rho_{i}\right) \sigma_{a} \varrho_{2}},
$$

It is worthy in mentioning that one of the eigenvalues of the above Jacobian $\mathcal{J}_{E_{0}}^{*}$ is 0 (simple eigenvalue) and the other four eigenvalues are negative or have negative real parts. Therefore applying the center manifold theorem [45] near $\beta_{s}^{*}$ and investigate the dynamics of the system (1).

We obtain the right eigenvector $w=\left[\begin{array}{llllll}w_{1} & w_{2} & w_{3} & w_{4} & w_{5} & w_{6}\end{array}\right]^{T}$ and the left eigenvector $v=\left[\begin{array}{llllll}v_{1} & v_{2} & v_{3} & v_{4} & v_{5} & v_{6}\end{array}\right]^{T}$ corresponding to the Jacobian $\mathcal{J}_{E_{0}}^{*}$. The components of the two eigenvectors $w$ and $v$ are given by

$$
\begin{aligned}
& w_{1}=-\frac{1}{\delta}\left[\frac{\beta_{s} \alpha_{a} S^{0} \rho_{i} \sigma_{a}}{\varrho_{2}}+\frac{\beta_{q}\left(1-\rho_{i}\right) \sigma_{a}}{\varrho_{3}}+\frac{\beta_{s} \alpha_{i} S^{0} \sigma_{a}\left(\beta_{i} \rho_{i} \varrho_{3}+\left(1-\rho_{i}\right) \alpha_{q} \varrho_{2}\right)}{\varrho_{2} \varrho_{3} \varrho_{4}}\right] w_{2}, \\
& w_{2}=w_{2}>0, w_{3}=\frac{\rho_{i} \sigma_{a}}{\varrho_{2}} w_{2}, w_{4}=\frac{\left(1-\rho_{i}\right) \sigma_{a}}{\varrho_{3}} w_{2}, \quad w_{5}=\frac{\sigma_{a}\left(\beta_{i} \rho_{i} \varrho_{3}+\left(1-\rho_{i}\right) \alpha_{q} \varrho_{2}\right)}{\varrho_{2} \varrho_{3} \varrho_{4}} w_{2}, \\
& w_{6}=\frac{1}{\delta}\left[\gamma_{a}+\frac{\rho_{i} \sigma_{a} \gamma_{i}}{\varrho_{2}}+\frac{\sigma_{a}\left(\beta_{i} \rho_{i} \varrho_{3}+\left(1-\rho_{i}\right) \alpha_{q} \gamma_{j} \varrho_{2}\right)}{\varrho_{2} \varrho_{3} \varrho_{4}}\right] w_{2}
\end{aligned}
$$


and

$v_{1}=0, v_{2}=v_{2}>0, v_{3}=\beta_{s} S^{0}\left[\alpha_{a}+\frac{\beta_{i} \alpha_{i}}{\varrho_{4}}\right] v_{2}, v_{4}=\frac{\beta_{s} S^{0} \alpha_{q} \alpha_{i}}{\varrho_{3} \varrho_{4}} v_{2}, v_{5}=\frac{\beta_{s} S^{0} \alpha_{i}}{\varrho_{4}} v_{2}$,

$v_{6}=0$.

Thus we can compute the constants $a$ and $b$ are as follows:

$$
\begin{aligned}
a & =\sum_{k, i, j=1}^{6} v_{k} w_{i} w_{j}\left[\frac{\partial^{2} \Theta_{k}}{\partial \chi_{i} \partial \chi_{j}}\left(E_{0}\right)\right]_{\beta_{s}=\beta_{s}^{*}} \\
\text { and } b & =\sum_{k, i=1}^{6} v_{k} w_{i}\left[\frac{\partial^{2} \Theta_{k}}{\partial \chi_{i} \partial \beta}\left(E_{0}\right)\right]_{\beta_{s}=\beta_{s}^{*}},
\end{aligned}
$$

Considering the non-zero second order partial derivatives measured by replacing the value at the disease-free equilibrium point $E^{*}$ at $\beta_{s}=\beta_{s}^{*}$, we have

$$
\begin{aligned}
a & =2 \beta_{s}^{*} v_{2} w_{1}\left(w_{2} \alpha_{a}+w_{3} \alpha_{i}+w_{5} \alpha_{j}\right)<0, \text { for } w_{1}<0, \\
\text { and } b & =v_{2}\left(w_{2} \alpha_{a}+w_{3} \alpha_{i}+w_{5} \alpha_{j}\right)>0,
\end{aligned}
$$

Therefore, $a<0$ and $b>0$ at bifurcation parameter $\beta_{s}=\beta_{s}^{*}$ a transcritical bifurcation happens at $R_{0}=1$. Therefore the unique interior fixed point $E^{*}\left(S^{*}, A^{*}, I^{*}, Q^{*}, J^{*}, R^{*}\right)$ is locally asymptotically stable if $R_{0}>1$ when $R_{0}$ is near to 1 .

\section{The optimal control problem}

In this section, we employ the theory of an optimal control, which is a powerful tool for theoretical analysis corresponding to the infectious diseases. By using this mathematical tool, we have produced conditions which will point out the appropriate steps to mitigate the span of novel coronavirus infection in the human population. Our main aim is to minimize the infected individuals and the hospitalized individuals by using the optimal intervention strategies 46. We performed the theoretical analysis as well as numerical illustrations to show how these control strategies make an evident on the transmission of SARS-CoV-2 virus and to minimize the disease burden and their implementations. The definition of the parameters and supposition leads to the model (1) which implies a coupled system of nonlinear differential equations with six state variables, that is, $S(t), A(t), I(t), Q(t), J(t)$ and $R(t)$. In this control problem, we have introduced three control variables $\eta_{i}(t)(i=1,2,3)$ that externally control the number of isolated or hospitalized cases and clinically ill or symptomatic infected cases over a specified time frame. 
3.1 Enhancing the response of susceptible individuals by modifying the isolation rate

Susceptible population begins to become aware of the diseases and their control strategies when they are given sufficient statistics and as a result the behavior of the population is changed. Due to the systematic news, the population has begun to wear masks, maintaining social distances with proper hygiene, remaining at quarantine and even following home isolation strategy. Thus, we have modified the isolation rate by introducing the control factor $\left(1-\xi_{1} \eta_{1}(t)\right)$ that quantifies the external attempt keep in preventing or controlling the of interplays between isolated $(J)$ and susceptible $(S)$ individuals. Therefore, in the term $\beta_{s}\left(\alpha_{j}\left(1-\xi_{1} \eta_{1}(t)\right) J\right) S$, the parameter $\beta_{s}$ represents the maximum transmission rate per isolated and per susceptible populations with $\left(1-\xi_{1} \eta_{1}(t)\right)$ representing the depletion in $\beta_{s}$ created by the outside isolation or hospitalized control; $\xi_{1}$ quantifies the usefulness of the control $\eta_{1}(t)$ (where $\left.\eta_{1}(t) \in[0,1]\right)$. The most expected framework is $\xi_{1} \eta_{1}(t) \approx 1$, that is, the condition when interplays between isolated and hospitalized populations are almost 'perfectly' avoided (prevented via successful isolation) decreasing the total transmission rate successfully to 0 . Here 0 describes no response and 1 describes fully response due to the given treatment. Therefore, this response intensity is explicitly associated with population's behavioral response. We have taken into account this response intensity $\eta_{1}(t)$ as a control variable. To increase the response among population via isolation or hospitalization, so that they alter their behavior and the cost will be associated as a nonlinear function of $\eta_{1}(t)$. We wish to obtain the optimal response for susceptible population via isolation or hospitalization.

\subsection{Better treatment strategy for infected individuals}

To provide the better treatment policy to the symptomatic infected individuals not only decreases the disease prevalence but also influences its development. At the present scenario in novel coronavirus, we assume that the treatment is accessible and is given to the clinically ill population. As the accessibility of resources associated to medical treatments can not be unbounded, thus in the model system (1), we introduce the treatment in the form $-\xi_{2} \eta_{2}(t) I$. Here, $\xi_{2}$ denotes the treatment rate with intensity rate $\eta_{2}(t)$. There are different costs associated such as medicines, kits, diagnosis, health protocols and other associated costs when medication is given. Therefore, we assume that the treatment intensity $\eta_{2}(t)$ as a control variable lies between 0 and 1 . The controlling attempt $\eta_{2}(t)$ changes the fraction of asymptotic infected cases $\xi_{2} I(t)$ obtaining antivirals per unit of time. 
3.3 Provided treatment to isolated individuals

In order to give the better treatment strategy against novel coronavirus to the isolated or hospitalized individuals at the very beginning of infection can lower the disease mortality. It also influences the development of the diseases. It can also be noted that the vaccine is accessible and given to the population who are admitted in the nursing home or hospitals in a limited amount. As the accessibility of resources associated to the medical treatments, financial crisis, disease diagnosis, etc., all the equipments are restricted. To keep this mind, we modified the model system (1) by introducing the treatment in the form $-\xi_{3} \eta_{3}(t) J$. Here, $\xi_{3}$ denotes the treatment rate with intensity rate $\eta_{3}(t)$. There are different cost related to the medicines, health problem, isolation, vaccination, medical kits, etc., at the time of medication period is need to be taken into account. Thus, we consider $\eta_{3}(t)$ as a control variable that lying between 0 and 1, where 0 represents no response and 1 represents full response after the treatment. The controlling effort $\eta_{3}(t)$ changes the fraction of isolated population $\left(\xi_{3} J(t)\right)$ obtaining the antiviral treatment per unit of time. Let us consider that the controls attain maximum per-capita effectiveness vaccination rates when the controls become 1 .

The main aim of this section is to obtain the optimal treatment strategy and optimal controls through clinically ill infected population and isolated individuals with minimum cost with the aid of vaccination. Thus, the admissible set for three control variables $\eta_{1}(t), \eta_{2}(t)$ and $\eta_{3}(t)$ is defined as follows:

$$
\mathcal{U}=\left\{\eta_{1}(t), \eta_{2}(t), \eta_{3}(t): 0 \leq \eta_{1}(t), \eta_{2}(t), \eta_{3}(t) \leq 1, t \in[0, T]\right\} .
$$

The following optimal control problem is investigated with the above discussed control strategies along with the objective functional $\mathcal{W}$ to be minimized:

$$
\begin{aligned}
\frac{d S}{d t} & =\Lambda_{s}-\beta_{s}\left(\alpha_{a} A+\alpha_{i} I+\alpha_{j}\left(1-\xi_{1} \eta_{1}(t)\right) J\right) S+\beta_{q} Q-\delta S, \\
\frac{d A}{d t} & =\beta_{s}\left(\alpha_{a} A+\alpha_{i} I+\alpha_{j}\left(1-\xi_{1} \eta_{1}(t)\right) J\right) S-\sigma_{a} A-\gamma_{a} A-\delta A, \\
\frac{d I}{d t} & =\rho_{i} \sigma_{a} A-\gamma_{i} I-\beta_{i} I-\xi_{2} \eta_{2}(t) I-\delta I, \\
\frac{d Q}{d t} & =\left(1-\rho_{i}\right) \sigma_{a} A-\alpha_{q} Q-\beta_{q} Q-\delta Q, \\
\frac{d J}{d t} & =\beta_{i} I+\alpha_{q} Q-\gamma_{j} J-\xi_{3} \eta_{3}(t) J-\delta J, \\
\frac{d R}{d t} & =\gamma_{a} A+\gamma_{i} I+\gamma_{j} J+\xi_{2} \eta_{2}(t) I+\xi_{3} \eta_{3}(t) J-\delta R,
\end{aligned}
$$

subject to minimize the objective functional

$\mathcal{W}\left(\eta_{1}(t), \eta_{2}(t), \eta_{3}(t)\right)=\int_{0}^{T}\left[\varphi_{1} I+\varphi_{2} J+\frac{1}{2}\left(\kappa_{1} \eta_{1}^{2}(t)+\kappa_{2} \eta_{2}^{2}(t)+\kappa_{3} \eta_{3}^{2}(t)\right)\right] d t$ 
with the initial conditions are stated in the equation (2). Here, the objective functional $\mathcal{W}$ represents the total incurred cost, that is, the sum of of the costs stated in the integrand:

$\mathcal{W}\left(S, A, I, Q, J, R, \eta_{1}(t), \eta_{2}(t), \eta_{3}(t)\right)=\varphi_{1} I+\varphi_{2} J+\frac{1}{2}\left(\kappa_{1} \eta_{1}^{2}(t)+\kappa_{2} \eta_{2}^{2}(t)+\kappa_{3} \eta_{3}^{2}(t)\right)$,

represents the current value of the cost at any time $t$. All the parameters $\varphi_{1}, \varphi_{2}, \kappa_{1}, \kappa_{2}$, and $\kappa_{3}$ are nonnegative. They are used as a weight constants and balancing the units of the integrand. Also they represents the measure of the relative costs of the control functions over $[0, T]$. The control functions $\eta_{1}^{*}(t), \eta_{2}^{*}(t)$ and $\eta_{3}^{*}(t)$ exists in the admissible control set $\mathcal{U}$ that mainly minimized the objective functional $\mathcal{W}$.

\subsection{Existence of optimal control}

In this subsection, the existence of an optimal control of (9) is analyzed. Then we employ the Pontryagin's-Maximum Principle [46] to characterize the optimal control. The Pontryagin's maximum principle is being utilized to get necessary conditions for the three controls. To prove the existence of an optimal control, we utilize the following theorem which assured that the existence of the controls $\eta_{1}^{*}, \eta_{2}^{*}$ and $\eta_{3}^{*}$ that minimizes the objective functional $\mathcal{W}$

Theorem 5 There exists optimal controls $\eta(t)=\left(\eta_{1}^{*}(t), \eta_{2}^{*}(t), \eta_{3}^{*}(t)\right)$ in $\mathcal{U}$ that minimizes the objective functional $\mathcal{W}\left(\eta_{1}(t), \eta_{2}(t), \eta_{3}(t)\right)$ corresponding to the control system (9)- (10).

Proof In the Section 2, we have already proved that all the solutions of (1) are non-negative and uniformly bounded for the given initial conditions (2). Non-negativity of the objective functional is obvious and the non-negativity implies the boundedness of the objective functional. Hence, minimizing the sequence of controls $\eta^{\tau}(t)=\left(\eta_{1}^{\tau}(t), \eta_{2}^{\tau}(t), \eta_{3}^{\tau}(t)\right) \in \mathcal{U}$ exists in such a way that

$$
\lim _{\tau \rightarrow \infty} \mathcal{W}\left(\eta^{\tau}(t)\right)=\inf _{\eta \in \mathcal{U}} \mathcal{W}(\eta(t))
$$

Therefore, the controls belonging to $\mathcal{U}$ are uniformly bounded in $L^{\infty}$ and consequently they are uniform bounded in $L^{2}([0, T])$. Since the space $L^{2}([0, T])$ is reflexive [4], then there exists $\eta^{*}(t)=\left(\eta_{1}^{*}(t), \eta_{2}^{*}(t), \eta_{3}^{*}(t)\right) \in \mathcal{U}$ a subsequence in such a way that

$\eta_{1}^{\tau}(t) \rightarrow \eta_{1}^{*}(t), \quad \eta_{2}^{\tau}(t) \rightarrow \eta_{2}^{*}(t), \quad \eta_{3}^{\tau}(t) \rightarrow \eta_{3}^{*}(t) \quad$ weakly in $L^{2}([0, T])$ as $\tau \rightarrow \infty$.

Then it is obvious that the state sequence $z^{\tau}=\left(S^{\tau}, A^{\tau}, I^{\tau}, Q^{\tau}, J^{\tau}, R^{\tau}\right)$ is uniformly bounded corresponding to $\eta^{\tau}(t)$. It can be seen that the uniformly boundedness of the right-hand side of the system (1) implies the uniformly boundedness of the derivatives for $z^{\tau}$ and equi-continuity of the corresponding state sequence $z^{\tau}$. Due to the Arzel-Ascoli Theorem, there exist $z^{\tau}=\left(S^{\tau}, A^{\tau}, I^{\tau}, Q^{\tau}, J^{\tau}, R^{\tau}\right)$ such that on a subsequence,

$$
z^{\tau} \rightarrow z^{*} \quad \text { uniformly on }[0, T]
$$


Next, we choose a proper subsequence passing through the limit of the system (1) and corresponding to the controls $\eta_{1}^{*}(t), \eta_{2}^{*}(t), \eta_{3}^{*}(t)$, we can achieve the state solution $z^{*}$. Then the lower semi-continuity of the $L^{2}$ - norm with respect to $L^{2}$ weak convergence implies that

$$
\begin{aligned}
\inf _{\eta \in \mathcal{U}} \mathcal{W}(\eta(t)) & =\lim _{\tau \rightarrow \infty} \mathcal{W}\left(\eta^{\tau}(t)\right) \geq \int_{0}^{T}\left(\varphi_{1} I+\varphi_{2} J\right) d t \\
& +\frac{1}{2} \int_{0}^{T}\left(\kappa_{1} \eta_{1}^{2}(t)+\kappa_{2} \eta_{2}^{2}(t)+\kappa_{3} \eta_{3}^{2}(t)\right) d t=\mathcal{W}\left(\eta^{*}(t)\right) .
\end{aligned}
$$

Therefore, $\eta^{*}(t)$ is an optimal control.

3.5 Characterization of optimal control

Since the existence of the controls are established for minimizing the objective functional 10 subject to the state equations $(9)$, then we employed Pontryagin's Maximum Principle [46] to obtain necessary conditions for the optimal control problem [4, 49.

Theorem 6 There exists optimal controls $\eta^{*}(t)=\left(\eta_{1}^{*}(t), \eta_{2}^{*}(t), \eta_{3}^{*}(t)\right) \in \mathcal{U}$ and the corresponding state solutions $z^{*}=\left(S^{*}, A^{*}, I^{*}, Q^{*}, J^{*}, R^{*}\right)$ of the system (9) that minimizes the objective functional $\mathcal{W}\left(\eta_{1}(t), \eta_{2}(t), \eta_{3}(t)\right)$, then there exist adjoint variables $\vartheta_{s}, \vartheta_{a}, \vartheta_{i}, \vartheta_{q}, \vartheta_{j}$, and $\vartheta_{r}$ satisfying the following canonical equations:

$$
\begin{aligned}
\vartheta_{s}^{\prime} & =-\left[\left(\vartheta_{a}-\vartheta_{s}\right) \beta_{s}\left(\alpha_{a} A+\alpha_{i} I+\alpha_{j}\left(1-\xi_{1} \eta_{1}(t)\right) J\right)-\vartheta_{s} \delta\right], \\
\vartheta_{a}^{\prime} & =-\left[\left(\vartheta_{a}-\vartheta_{s}\right) \beta_{s} \alpha_{a} S-\vartheta_{a}\left(\sigma_{a}+\gamma_{a}+\delta\right)+\vartheta_{i} \rho_{i} \sigma_{a}+\vartheta_{q}\left(1-\rho_{i}\right) \sigma_{a}+\vartheta_{r} \gamma_{a}\right], \\
\vartheta_{i}^{\prime} & =-\left[\varphi_{1}+\left(\vartheta_{a}-\vartheta_{s}\right) \beta_{s} \alpha_{i} S-\vartheta_{i}\left(\gamma_{i}+\beta_{i}+\delta+\xi_{2} \eta_{2}(t)\right)+\vartheta_{j} \beta_{i}+\vartheta_{r}\left(\gamma_{i}+\xi_{2} \eta_{2}(t)\right)\right], \\
\vartheta_{q}^{\prime} & =-\left[\vartheta_{s} \beta_{q}-\vartheta_{q}\left(\alpha_{q}+\beta_{q}+\delta\right)+\vartheta_{j} \alpha_{q}\right], \\
\vartheta_{j}^{\prime} & =-\left[\varphi_{2}+\left(\vartheta_{a}-\vartheta_{s}\right)\left(1-\xi_{1} \eta_{1}(t)\right) \beta_{s} \alpha_{j} S-\vartheta_{j}\left(\gamma_{j}+\delta+\xi_{3} \eta_{3}(t)\right)+\vartheta_{r}\left(\gamma_{j}+\xi_{3} \eta_{3}(t)\right)\right], \\
\vartheta_{r}^{\prime} & =-\left[-\vartheta_{r} \delta\right],
\end{aligned}
$$

with the transversality conditions:

$$
\vartheta_{s}(T)=0, \vartheta_{a}(T)=0, \vartheta_{i}(T)=0, \vartheta_{q}(T)=0, \vartheta_{j}(T)=0, \vartheta_{r}(T)=0 .
$$

Moreover, the corresponding optimal controls $\eta^{*}(t)=\left(\eta_{1}^{*}(t), \eta_{2}^{*}(t), \eta_{3}^{*}(t)\right)$ are represented by

$$
\begin{aligned}
& \eta_{1}^{*}(t)=\min \left\{\max \left\{0, \frac{\left(\vartheta_{a}-\vartheta_{s}\right) \beta_{s} \xi_{1} \alpha_{j} S J}{\kappa_{1}}\right\}, 1\right\}, \\
& \eta_{2}^{*}(t)=\min \left\{\max \left\{0, \frac{\left(\vartheta_{i}-\vartheta_{r}\right) \xi_{2} I}{\kappa_{2}}\right\}, 1\right\}, \\
& \eta_{3}^{*}(t)=\min \left\{\max \left\{0, \frac{\left(\vartheta_{j}-\vartheta_{r}\right) \xi_{3} J}{\kappa_{3}}\right\}, 1\right\} .
\end{aligned}
$$


Proof We investigate necessary conditions for the control functions with the aid of the Pontryagin's Maximum Principle for the model system (9). In order to do this, we construct the Hamiltonian $\mathcal{H}$ which is defined for all $t \in[0, T]$ as follows:

$$
\begin{aligned}
\mathcal{H}= & \varphi_{1} I+\varphi_{2} J+\frac{1}{2}\left(\kappa_{1} \eta_{1}^{2}(t)+\kappa_{2} \eta_{2}^{2}(t)+\kappa_{3} \eta_{3}^{2}(t)\right) \\
& +\vartheta_{s}\left[\Lambda_{s}-\beta_{s}\left(\alpha_{a} A+\alpha_{i} I+\alpha_{j}\left(1-\xi_{1} \eta_{1}(t)\right) J\right) S+\beta_{q} Q-\delta S\right] \\
& +\vartheta_{a}\left[\beta_{s}\left(\alpha_{a} A+\alpha_{i} I+\alpha_{j}\left(1-\xi_{1} \eta_{1}(t)\right) J\right) S-\sigma_{a} A-\gamma_{a} A-\delta A\right] \\
& +\vartheta_{i}\left[\rho_{i} \sigma_{a} A-\gamma_{i} I-\beta_{i} I-\xi_{2} \eta_{2}(t) I-\delta I\right] \\
& +\vartheta_{q}\left[\left(1-\rho_{i}\right) \sigma_{a} A-\alpha_{q} Q-\beta_{q} Q-\delta Q\right] \\
& +\vartheta_{j}\left[\beta_{i} I+\alpha_{q} Q-\gamma_{j} J-\xi_{3} \eta_{3}(t) J-\delta J\right] \\
& +\vartheta_{r}\left[\gamma_{a} A+\gamma_{i} I+\gamma_{j} J+\xi_{2} \eta_{2}(t) I+\xi_{3} \eta_{3}(t) J-\delta R\right]
\end{aligned}
$$

where $\vartheta_{s}, \vartheta_{a}, \vartheta_{i}, \vartheta_{q}, \vartheta_{j}$, and $\vartheta_{r}$ are the co-states or adjoint variables. With the aid of Pontryagin's Maximum Principle, we obtain the minimized Hamiltonian which minimizes the objective functional $\mathcal{W}\left(\eta_{1}(t), \eta_{2}(t), \eta_{3}(t)\right)$. Pontryagin's Maximum Principle plays an important role in adjoining the objective functional with the state variables including co-states or adjoint variables. Due to Pontryagin's Maximum Principle, there exist co-states $\vartheta_{s}^{\prime}, \vartheta_{a}^{\prime}, \vartheta_{i}^{\prime}, \vartheta_{q}^{\prime}$, $\vartheta_{j}^{\prime}$ and $\vartheta_{r}^{\prime}$ that satisfies the following canonical expressions

$$
\begin{aligned}
& \vartheta_{s}^{\prime}=-\frac{\partial \mathcal{H}}{\partial S}, \quad \vartheta_{a}^{\prime}=-\frac{\partial \mathcal{H}}{\partial A}, \quad \vartheta_{i}^{\prime}=-\frac{\partial \mathcal{H}}{\partial I}, \\
& \vartheta_{q}^{\prime}=-\frac{\partial \mathcal{H}}{\partial Q}, \quad \vartheta_{j}^{\prime}=-\frac{\partial \mathcal{H}}{\partial J}, \quad \vartheta_{r}^{\prime}=-\frac{\partial \mathcal{H}}{\partial R},
\end{aligned}
$$

with the transversality conditions: $\vartheta_{s}(T)=0, \vartheta_{a}(T)=0, \vartheta_{i}(T)=0, \vartheta_{q}(T)=$ $0, \vartheta_{j}(T)=0$, and $\vartheta_{r}(T)=0$. Now, we get the characterization of optimal controls are given by

$$
\frac{\partial \mathcal{H}}{\partial \eta_{1}(t)}=0, \quad \frac{\partial \mathcal{H}}{\partial \eta_{2}(t)}=0, \quad \frac{\partial \mathcal{H}}{\partial \eta_{3}(t)}=0
$$

From $\partial \mathcal{H} / \partial \eta_{1}(t)=0, \partial \mathcal{H} / \partial \eta_{2}(t)=0$, and $\partial \mathcal{H} / \partial \eta_{3}(t)=0$, we get

$$
\begin{array}{ll}
\eta_{1}(t)=\frac{\left(\vartheta_{a}-\vartheta_{s}\right) \beta_{s} \xi_{1} \alpha_{j} S J}{\kappa_{1}} & \text { at } \eta_{1}(t)=\eta_{1}^{*}(t), \\
\eta_{2}(t)=\frac{\left(\vartheta_{i}-\vartheta_{r}\right) \xi_{2} I}{\kappa_{2}} & \text { at } \eta_{2}(t)=\eta_{2}^{*}(t), \\
\eta_{2}(t)=\frac{\left(\vartheta_{j}-\vartheta_{r}\right) \xi_{3} J}{\kappa_{3}} & \text { at } \eta_{3}(t)=\eta_{3}^{*}(t) .
\end{array}
$$


By considering the upper and lower bounds for $\eta_{1}(t), \eta_{2}(t)$, and $\eta_{3}(t)$, we have following the characterization of the optimal controls:

$$
\begin{gathered}
\eta_{1}^{*}(t)=\min \left\{\max \left\{0, \frac{\left(\vartheta_{a}-\vartheta_{s}\right) \beta_{s} \xi_{1} \alpha_{j} S J}{\kappa_{1}}\right\}, 1\right\}, \\
\eta_{2}^{*}(t)=\min \left\{\max \left\{0, \frac{\left(\vartheta_{i}-\vartheta_{r}\right) \xi_{2} I}{\kappa_{2}}\right\}, 1\right\}, \\
\eta_{3}^{*}(t)=\min \left\{\max \left\{0, \frac{\left(\vartheta_{j}-\vartheta_{r}\right) \xi_{3} J}{\kappa_{3}}\right\}, 1\right\} .
\end{gathered}
$$

It can also be noted that

$$
\frac{\partial^{2} \mathcal{H}}{\partial \eta_{1}^{2}(t)}=\kappa_{1}>0, \quad \frac{\partial^{2} \mathcal{H}}{\partial \eta_{2}^{2}(t)}=\kappa_{2}>0, \quad \frac{\partial^{2} \mathcal{H}}{\partial \eta_{3}^{2}(t)}=\kappa_{3}>0,
$$

which assured that our optimal controls minimize the Hamiltonian.

Now we derive the corresponding optimality system utilizing the optimal controls $\eta^{*}(t)=\left(\eta_{1}^{*}(t), \eta_{2}^{*}(t), \eta_{3}^{*}(t)\right)$ that has already been characterized in the above. The optimality system that minimized Hamiltonian $\mathcal{H}$ at $\left(S^{*}, A^{*}, I^{*}\right.$, $\left.Q^{*}, J^{*}, R^{*}, \eta_{1}^{*}, \eta_{2}^{*}, \eta_{3}^{*}, \vartheta_{s}, \vartheta_{a}, \vartheta_{i}, \vartheta_{q}, \vartheta_{j}, \vartheta_{r}\right)$ is given as follows:

$$
\begin{aligned}
\frac{d S}{d t} & =\Lambda_{s}-\beta_{s}\left(\alpha_{a} A^{*}+\alpha_{i} I^{*}+\alpha_{j}\left(1-\xi_{1} \eta_{1}^{*}(t)\right) J^{*}\right) S^{*}+\beta_{q} Q^{*}-\delta S^{*} \\
\frac{d A}{d t} & =\beta_{s}\left(\alpha_{a} A^{*}+\alpha_{i} I^{*}+\alpha_{j}\left(1-\xi_{1} \eta_{1}^{*}(t)\right) J^{*}\right) S^{*}-\sigma_{a} A^{*}-\gamma_{a} A^{*}-\delta A^{*} \\
\frac{d I}{d t} & =\rho_{i} \sigma_{a} A^{*}-\gamma_{i} I^{*}-\beta_{i} I^{*}-\xi_{2} \eta_{2}^{*}(t) I^{*}-\delta I^{*} \\
\frac{d Q}{d t} & =\left(1-\rho_{i}\right) \sigma_{a} A^{*}-\alpha_{q} Q^{*}-\beta_{q} Q^{*}-\delta Q^{*} \\
\frac{d J}{d t} & =\beta_{i} I^{*}+\alpha_{q} Q^{*}-\gamma_{j} J^{*}-\xi_{3} \eta_{3}^{*}(t) J^{*}-\delta J^{*} \\
\frac{d R}{d t} & =\gamma_{a} A^{*}+\gamma_{i} I^{*}+\gamma_{j} J^{*}+\xi_{2} \eta_{2}^{*}(t) I^{*}+\xi_{3} \eta_{3}^{*}(t) J^{*}-\delta R^{*}
\end{aligned}
$$

with initial values: $S^{*}(0) \geq 0, A^{*}(0) \geq 0, I^{*}(0) \geq 0, Q^{*}(0) \geq 0, J^{*}(0) \geq 0$, $R^{*}(0) \geq 0$ and the corresponding co-states are

$$
\begin{aligned}
\vartheta_{s}^{\prime} & =-\left[\left(\vartheta_{a}-\vartheta_{s}\right) \beta_{s}\left(\alpha_{a} A^{*}+\alpha_{i} I^{*}+\alpha_{j}\left(1-\xi_{1} \eta_{1}^{*}(t)\right) J^{*}\right)-\vartheta_{s} \delta\right], \\
\vartheta_{a}^{\prime} & =-\left[\left(\vartheta_{a}-\vartheta_{s}\right) \beta_{s} \alpha_{a} S^{*}-\vartheta_{a}\left(\sigma_{a}+\gamma_{a}+\delta\right)+\vartheta_{i} \rho_{i} \sigma_{a}+\vartheta_{q}\left(1-\rho_{i}\right) \sigma_{a}+\vartheta_{r} \gamma_{a}\right], \\
\vartheta_{i}^{\prime} & =-\left[\varphi_{1}+\left(\vartheta_{a}-\vartheta_{s}\right) \beta_{s} \alpha_{i} S^{*}-\vartheta_{i}\left(\gamma_{i}+\beta_{i}+\delta+\xi_{2} \eta_{2}^{*}(t)\right)+\vartheta_{j} \beta_{i}+\vartheta_{r}\left(\gamma_{i}+\xi_{2} \eta_{2}^{*}(t)\right)\right], \\
\vartheta_{q}^{\prime} & =-\left[\vartheta_{s} \beta_{q}-\vartheta_{q}\left(\alpha_{q}+\beta_{q}+\delta\right)+\vartheta_{j} \alpha_{q}\right], \\
\vartheta_{j}^{\prime} & =-\left[\varphi_{2}+\left(\vartheta_{a}-\vartheta_{s}\right)\left(1-\xi_{1} \eta_{1}^{*}(t)\right) \beta_{s} \alpha_{j} S-\vartheta_{j}\left(\gamma_{j}+\delta+\xi_{3} \eta_{3}^{*}(t)\right)+\vartheta_{r}\left(\gamma_{j}+\xi_{3} \eta_{3}^{*}(t)\right)\right], \\
\vartheta_{r}^{\prime} & =-\left[-\vartheta_{r} \delta\right],
\end{aligned}
$$


with the transversality conditions are $\vartheta_{s}(T)=0 *, \vartheta_{a}(T)=0, \vartheta_{i}(T)=0$, $\vartheta_{q}(T)=0, \vartheta_{j}(T)=0, \vartheta_{r}(T)=0$, and the three controls $\eta_{1}^{*}, \eta_{2}^{*}$ and $\eta_{3}^{*}$ are the same as 12 - 14 . Hence the proof of the theorem.

Table 2 Description of system parameter values used for novel coronavirus model system (1).

\begin{tabular}{|c|c|c|c|}
\hline Parameter & Description & Values (Unit) & Source \\
\hline$\Lambda_{s}$ & inflow rate of susceptible individual & $\begin{array}{l}1.6 \times 10^{6} \\
\text { person day }\end{array}$ & 27 \\
\hline$\beta_{s}$ & disease transmission rate & $10^{-7}$ day $^{-1}$ & Estimated \\
\hline$\alpha_{a}$ & modification factor for asymptomatic population & 0.001 & Estimated \\
\hline$\alpha_{i}$ & modification factor for symptomatic population & 0.0001 & 50 \\
\hline$\alpha_{j}$ & adjustment factor for isolated individuals & 0.036 & 50 \\
\hline$\beta_{q}$ & the rate that quarantined becomes susceptible & 0.0135 day $^{-1}$ & Fit to data \\
\hline$\delta$ & natural mortality rate of all the populations & 0.005 day $^{-1}$ & Estimated \\
\hline$\gamma_{a}$ & $\begin{array}{l}\text { asymptomatic individuals recovered from the dis- } \\
\text { ease }\end{array}$ & 0.0066 day $^{-1}$ & Estimated \\
\hline$\sigma_{a}$ & progression rate of infectious & 0.014 day $^{-1}$ & Estimated \\
\hline$\rho_{i}$ & $\begin{array}{l}\text { fraction of asymptotic individuals showing clinical } \\
\text { symptoms }\end{array}$ & 0.031 day $^{-1}$ & Estimated \\
\hline$\gamma_{i}$ & infected population progress to the recovered class & $0.002 \mathrm{day}^{-1}$ & 24 \\
\hline$\beta_{i}$ & infected population progress to the isolated class & 0.653 day $^{-1}$ & 23 \\
\hline$\alpha_{q}$ & rate at which quarantined becomes isolated & 0.337 day $^{-1}$ & Assumed \\
\hline$\gamma_{j}$ & isolated population progress to the recovered class & 0.997 day $^{-1}$ & Fit to data \\
\hline
\end{tabular}

\section{Model calibration and data source}

In this Section, we validate our theoretical analysis based on the numerical solutions with real data from India. In order to do this, we estimate the most sensitive parameters for our SAIQJR model.

\subsection{Sensitivity analysis}

We have calibrated our novel coronavirus model (1) by using the daily confirmed new SARS-CoV-2 viruses and cumulative confirmed SARS-CoV-2 viruses for India. We have taken data for novel coronavirus diseases from World Health Organization (WHO) registered cases for the time period February 01, 2021 to May 21, 2021 [51]. Our proposed model (1) has a system of 6 coupled ordinary differential equations with 14 parameters, among them six key parameters have been estimated by using semi-relative sensitivity analysis [52], namely $\beta_{s}, \alpha_{a}$, $\delta, \sigma_{a}, \rho_{i}$ and $\gamma_{a}$ for the novel coronavirus model (1). These six parameters value and the initial individuals size plays a critical role in the model analysis.

Sensitivity analysis depicts the significance of parameter alters in the model output. Due to the lines of Martin Fink [53], we draw the sensitivity graph by 

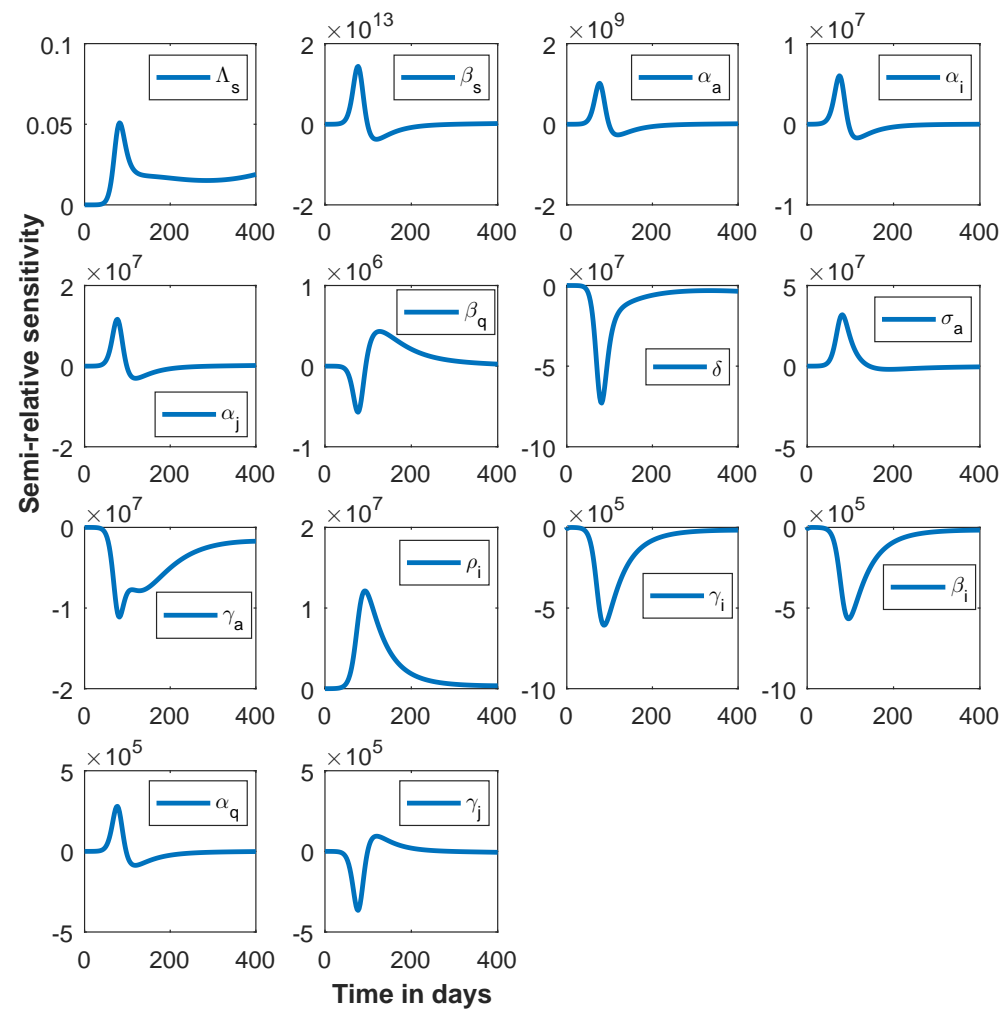

Fig. 2 The figure represents the semi-relative sensitivities of the system parameters by using automatic differentiation. From the figures it can be observed that the parameters $\beta_{s}$, $\alpha_{a}, \delta, \sigma_{a}, \rho_{i}, \gamma_{a}, \alpha_{j}$ are most effective with respect to the symptomatic infected individuals. The biologically realistic time window is $[0,400]$.

utilizing the code myAD, established by Fink [53] (see the Fig. 22). To quantify the sensitivities of the parameters from the Fig. 2, we calculate the sensitivity coefficient by calculating $L^{2}$-norm that can be defined as follows:

$$
C_{i j}=\left\|\frac{\partial x_{i}}{\partial q_{j}} \frac{q_{j}}{\max x_{i}}\right\|_{2}^{2}=\int_{t_{0}}^{t_{f}}\left|\frac{\partial x_{i}}{\partial q_{j}} \frac{q_{j}}{\max x_{i}}\right|^{2} d t .
$$

From the computation of $L^{2}$-norm it is clear that the most effective parameters are $\beta_{s}, \alpha_{a}, \delta, \sigma_{a}, \rho_{i}$ and $\gamma_{a}$ in descending order (see the Fig. 3) by comparing and ranking the sensitivity function that represents the other model parameters are less sensitive with respect to the clinically ill or symptomatic infected population, thus they will affect the dynamics of novel coronavirus a little.

A parameter is known as practically identifiable if an individual estimate can be determined from various initial conditions utilizing the available data. 


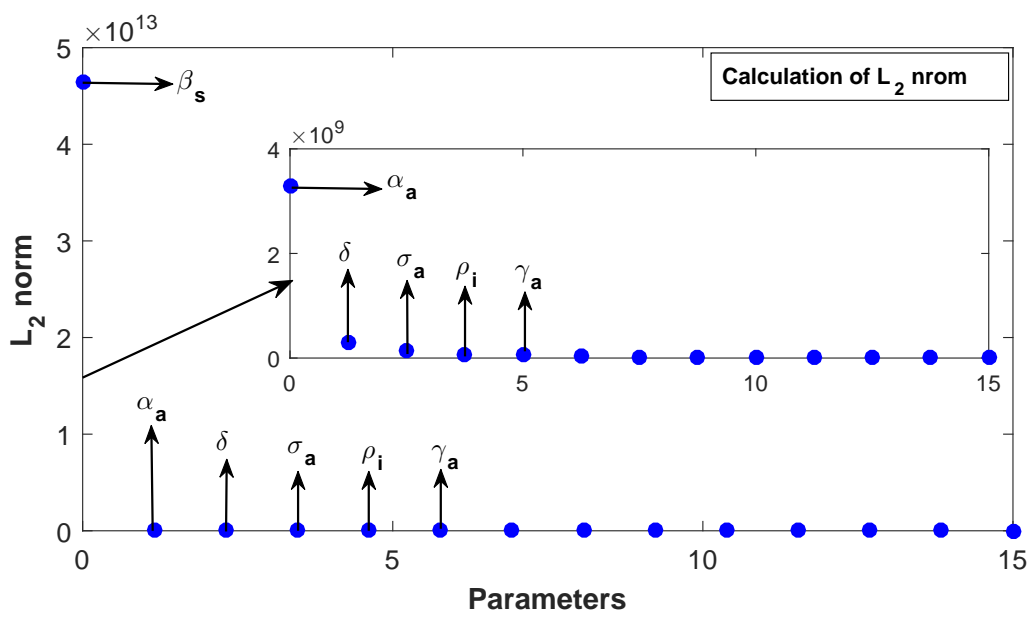

Fig. 3 The figure describes the quantification of semi-relative sensitivity analysis by computing sensitivity coefficient using $L_{2}$ norm.

We consider Fisher's information matrix $F=S^{T} S$, where $S$ represents the normalized sensitivity matrix calculated by automatic differentiation [53. It can be noticed that $m$ parameters are said to be locally identifiable iff the column rank of the matrix $S=m$, or identically $\operatorname{det}\left(S^{T} S\right) \neq 0$. After that, we execute $Q R$ factorization method with column pivoting in MATLAB routine $q r,[Q, R, P]=q r(F)$. This procedure find out matrix $P$ in such a way that $F P=Q R(Q R$ is the factorization of $F P)$. This indices in first $k$ columns of $P$ recognize $k$ parameters are most estimable. In our study, $\beta_{s}, \alpha_{a}, \delta, \sigma_{a}, \rho_{i}$ and $\gamma_{a}$ are the model parameters that are most estimable from the novel coronavirus data. We have estimated the most sensitive parameters by using Least Square Method, after identifying them. The values of these parameters are provided in Table 2. We have estimated the data for 110 days (from February 01, 2021 to May 21, 2021). Model fitting is shown in the Fig. 4

\subsection{Model simulation without control strategy}

We performed the numerical simulations in two folds, namely without administration of optimal control and with the administration of optimal control theory. For the validation of our theoretical analysis, we conduct some numerical simulations for the model parameters that are specified in the Table 2 Due to the present situation of the COVID-19 pandemic in India, the newly infected population per unit time is represented by $\beta S I \equiv \beta_{s}\left(\alpha_{a} A+\alpha_{i} I+\alpha_{j} J\right)$. The total population in India in August 2021 is approximately 138 million and according to the data from World Health Organization (WHO) 51 the daily confirmed number of infected cases on February 01, 2021 is 11427. Thus, we 

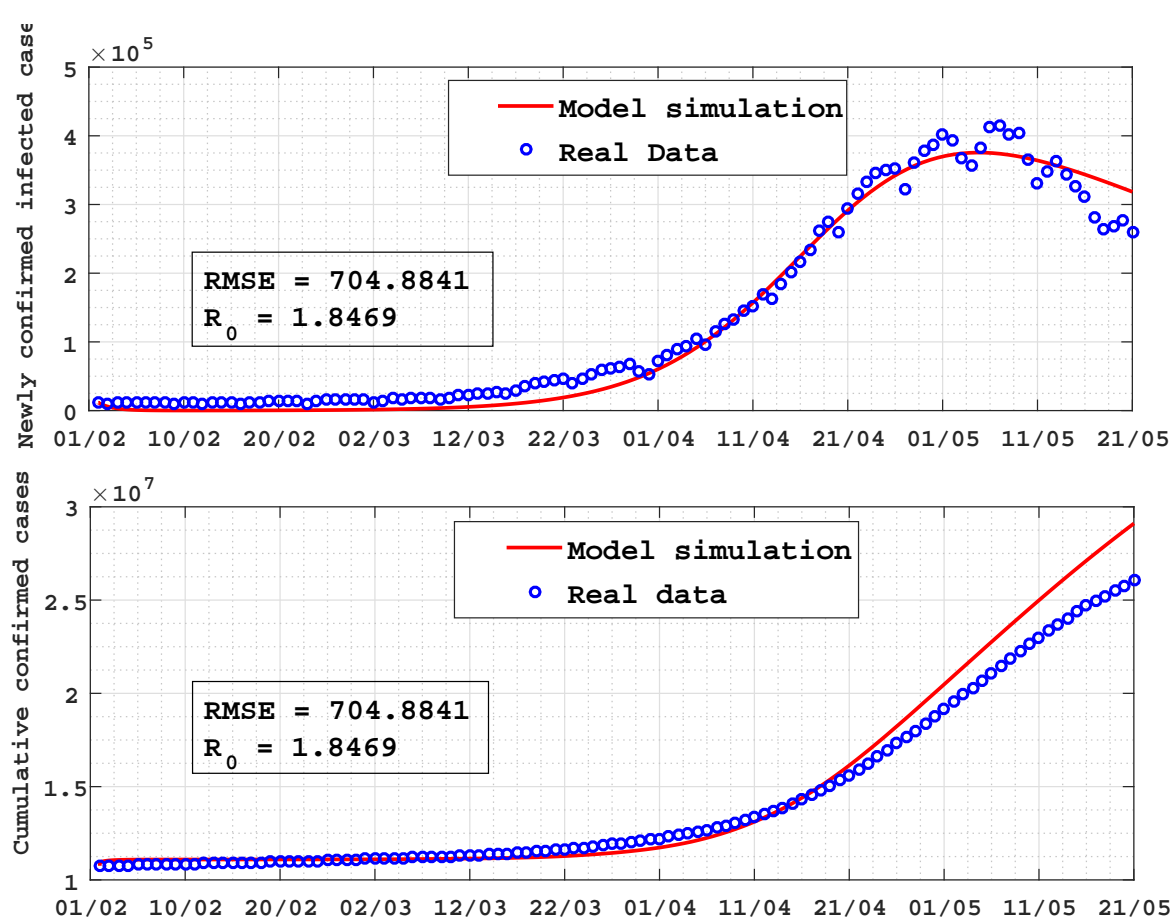

Fig. 4 Parameter estimation for the novel coronavirus model system 11 based on data from India. The SAIQJR model fitted with the real data for the daily new confirmed COVID-19 cases and the cumulative confirmed COVID-19 cases of India for the time period February 01, 2021 to May 21, 2021 (110 days). Real data are shown in blue circles whereas the red curve represents the best fitting curve of the system (1). Parameters are used for numerical solution and specified in the Table 2 . The initial size of the individuals used for numerical simulation are given in the text.

have used the following set of the initial size of the populations:

$$
\begin{gathered}
\mathcal{S} \equiv\{S(0)=1380004385, A(0)=5000, I(0)=11427, Q(0)=1, \\
J(0)=10, R(0)=1\} .
\end{gathered}
$$

The figures (Fig. 6(a)-(d)) represent the contour plots demonstrate the dependence of the basic reproduction number $R_{0}$ with reference to $\beta_{s}$ (disease transmission rate) and $\sigma_{a}$ (progression rate of infected individuals); $\alpha_{a}$ (adjustment factor for clinically ill population) and $\sigma_{a} ; \alpha_{j}$ (fraction of isolated or hospitalized individuals) and $\sigma_{a} ; \alpha_{a}$ and $\beta_{i}$ (infected individuals progress to isolated class) for the Republic of India. From the Fig. 6(a), we noticed that for an increasing value of disease transmission rate $\beta_{s}$ and $\sigma_{a}$, the value of the basic reproduction number $R_{0}$ increases. Similar phenomena has been noticed from the Fig. 6((b)-(d)). Thus from the Fig. 6(a), we can conclude that in order to mitigate the transmission of SARS-CoV-2, the basic reproduction 


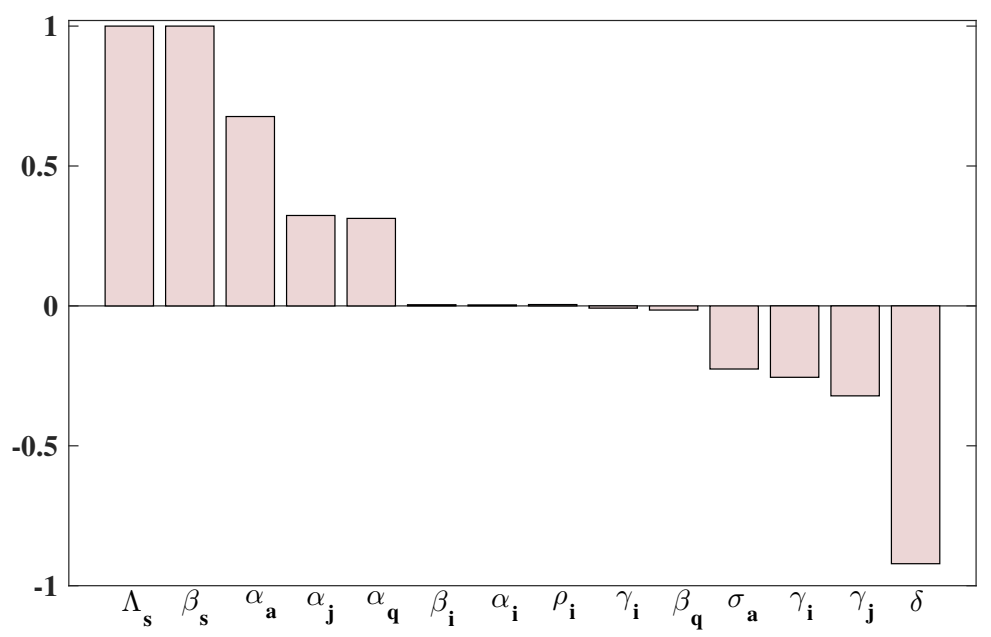

Fig. 5 Tornado plot for normalized forward sensitivity indices of the model parameters related with basic reproduction number $R_{0}$ are listed in the Table 1 indicating that the most sensitive parameters are $\beta_{s}, \Lambda_{s}$ and $\delta$.

number $R_{0}$ need to be maintained and that can be possible by maintaining the social distancing as well as contact tracing.

As we are familiar with that the numerical value of $R_{0}$ describes the behavior of the infectious diseases. From Table1 \& Fig. 5. Fig.6((a)-(d)), we can see that parameters with non-positive indices can be improved then the magnitude of $R_{0}$ would be diminished substantially. The model parameters namely $\beta_{s}$, $\Lambda_{s}$ and $\alpha_{a}$ has nonnegative indices and thus mitigation of these three model parameters can control the outbreak of the COVID-19 diseases. Moreover, from the sensitivity indices, we can observe that three parameters associated to susceptible population namely $\beta_{s}, \Lambda_{s}$ and $\alpha_{a}$ are the most effective parameters and we can restrict positively these three parameters. Therefore, we may deduce the most favorable method to maintain the COVID-19 diseases is to decrease the value of the parameters.

To better understand the threshold parameter $R_{0}$, we have drawn a surface plot for $R_{0}$ with reference to $\beta_{s}$ (rate of disease transmission) and $\gamma_{a}$ (rate by which asymptomatic populations become recovered) and the other parameters are defined in the Table 2. The expression for basic reproduction number is defined in 4 and we note that $\frac{d R_{0}}{d \beta_{s}}>0$ and $\frac{d R_{0}}{d \gamma_{a}}<0$. If we increase $\beta_{s}$, the basic reproduction number $R_{0}$ will increase and cross the value $R_{0}=1$, thus leading to the substantial outbreak of the SARS-CoV-2 virus. Moreover, when the recovery rate $\gamma_{a}$ of asymptomatic individuals increases, the basic reproduction number $R_{0}$ will decrease and if $R_{0}$ becomes lower than 1 , the infected populations will no longer persist. The surface plot shows in the Fig. 7 represents the behavior of $R_{0}$ in the range of $\beta_{s}$ and $\gamma_{a}$. Therefor, our model 

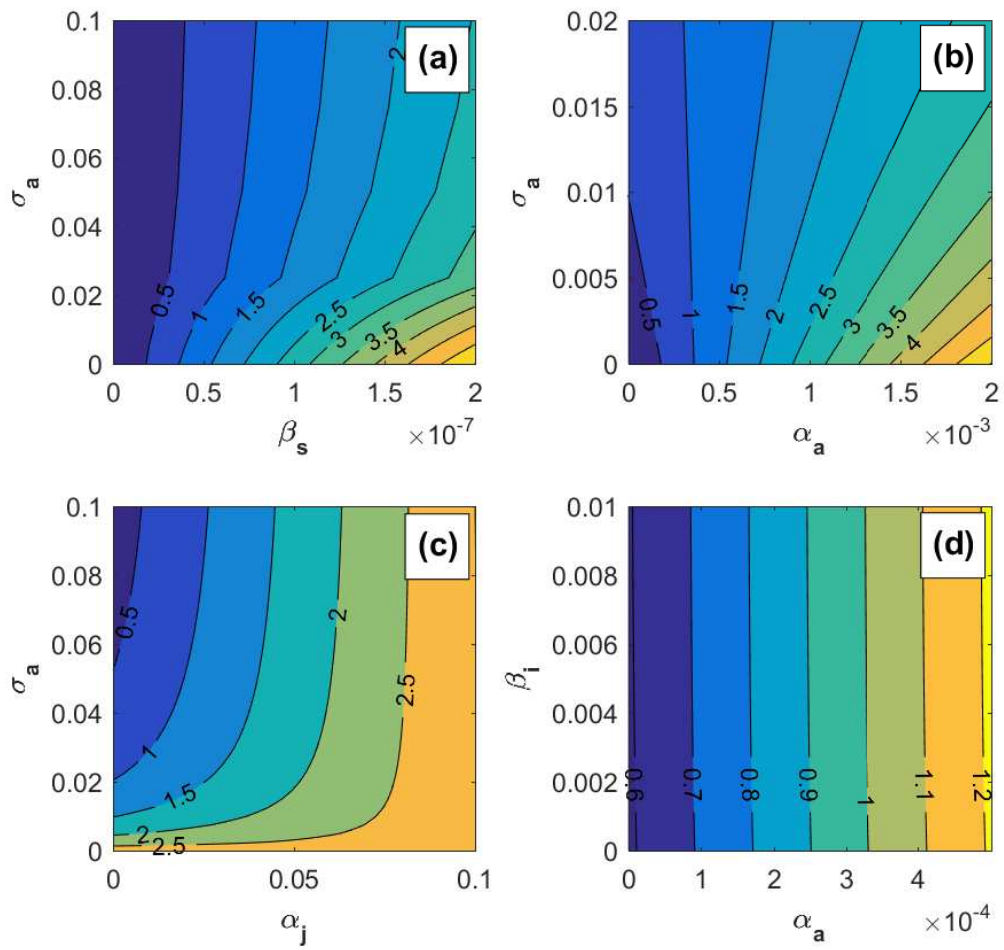

Fig. 6 Contour plots of the basic reproduction number $R_{0}$ for the case of India. (a) Contour plot of $R_{0}$ as a function of $\beta_{s}$ and $\sigma_{a}$; (b) Contour plot of $R_{0}$ as a function of $\alpha_{a}$ and $\sigma_{a}$; (c) Contour plot of $R_{0}$ as a function of $\alpha_{j}$ and $\sigma_{a}$; (d) Contour plot of $R_{0}$ as a function of $\alpha_{a}$ and $\beta_{i}$. All parameter values other than (a) $\beta_{s}$ and $\sigma_{a}$; (b) $\alpha_{a}$ and $\sigma_{a} ;$ (c) $\alpha_{j}$ and $\sigma_{a}$;

(d) $\alpha_{a}$ and $\beta_{i}$ are specified in the Table 2

illustration proposed that the recovery rate $\gamma_{a}$ of asymptomatic individuals aids to eliminate the novel coronavirus by decreasing the basic reproduction number $R_{0}$. Also, our simulation suggest that the reproduction number can be maintained by decreasing the value of the disease transmission rate $\beta_{s}$ and that can be achievable by maintaining social distancing and contact tracing.

\subsection{Model simulation with control strategy}

Now, we introduce additional recovery through the medication by using the theory of optimal control problem. In the model system (9), we have introduced three different control policies to mitigate the transmission of novel coronavirus burden and to minimize the total cost by obtaining the optimal control paths. We performed numerical simulations to investigate these outcomes and also to observe the influence of different parameters and intervention strategies on the 


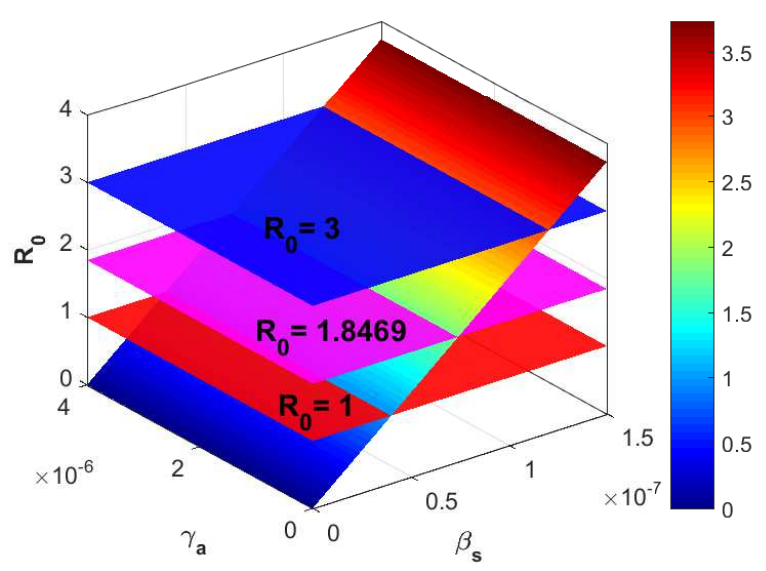

Fig. 7 The diagram shows the surface plot of $R_{0}$ as a function of $\beta_{s}$ (disease transmission rate) and $\gamma_{a}$ (the rate at which asymptomatic individuals become recovered). All parameter values other than $\beta_{s}$ and $\gamma_{a}$ are given in the Table 2

transmission dynamics of COVID-19. To investigate the numerical simulation of the objective functional $(10)$ subject to the coupled system of ordinary differential equations (9), we utilize fourth-order Runge-Kutta method with the aid of MATLAB. We solve the co-states (11) by using backward Runge-Kutta process by employing the solutions of $(9)$ with the transversality conditions defined in the Theorem 6. After that these controls are upgraded for the optimality utilizing the Hamiltonian of the optimal system. To do this, we used the steepest descent method of optimization that has been studied in [48, 49. The time frame of investigation and implementation of the optimal control strategies is considered to be 400 days. To solve the controlled system and the corresponding adjoint system, we use the parameters set defined in the Table 2 .

To analyze the impact of optimal treatment policies for the transmission dynamics of novel coronavirus, we employ different control policies for the disease compartments, namely asymptomatic population, clinically ill population, quarantine populations and the isolated individuals. In general, high basic reproduction number $\left(R_{0}>1.8469\right)$ create epidemics with high epidemic peak. The Fig. $8((\mathrm{a})-(\mathrm{d}))$ represents the impact of each treatment strategies on the disease state variables in presence of optimal control as well as without optimal controls when $R_{0}=1.8469$. These plots demonstrates the asymptomatic cases, daily number of infected cases, quarantine individuals and the isolated or hospitalized cases under no control $\left(\eta_{1}(t)=\eta_{2}(t)=\eta_{3}(t)=0\right)$, with the combination of three different controls $\left(\eta_{1}(t), \eta_{2}(t), \eta_{3}(t)\right)$ and for single controls $\eta_{i}(t)$ where $i=1,2,3$. The solid red curves for epidemic (without administration of intervention strategies) are exhibited to highlight the difference from those created via implementation of optimal treatment polices. The solid blue epidemic curves (with the implementation of three controls) are shown the 

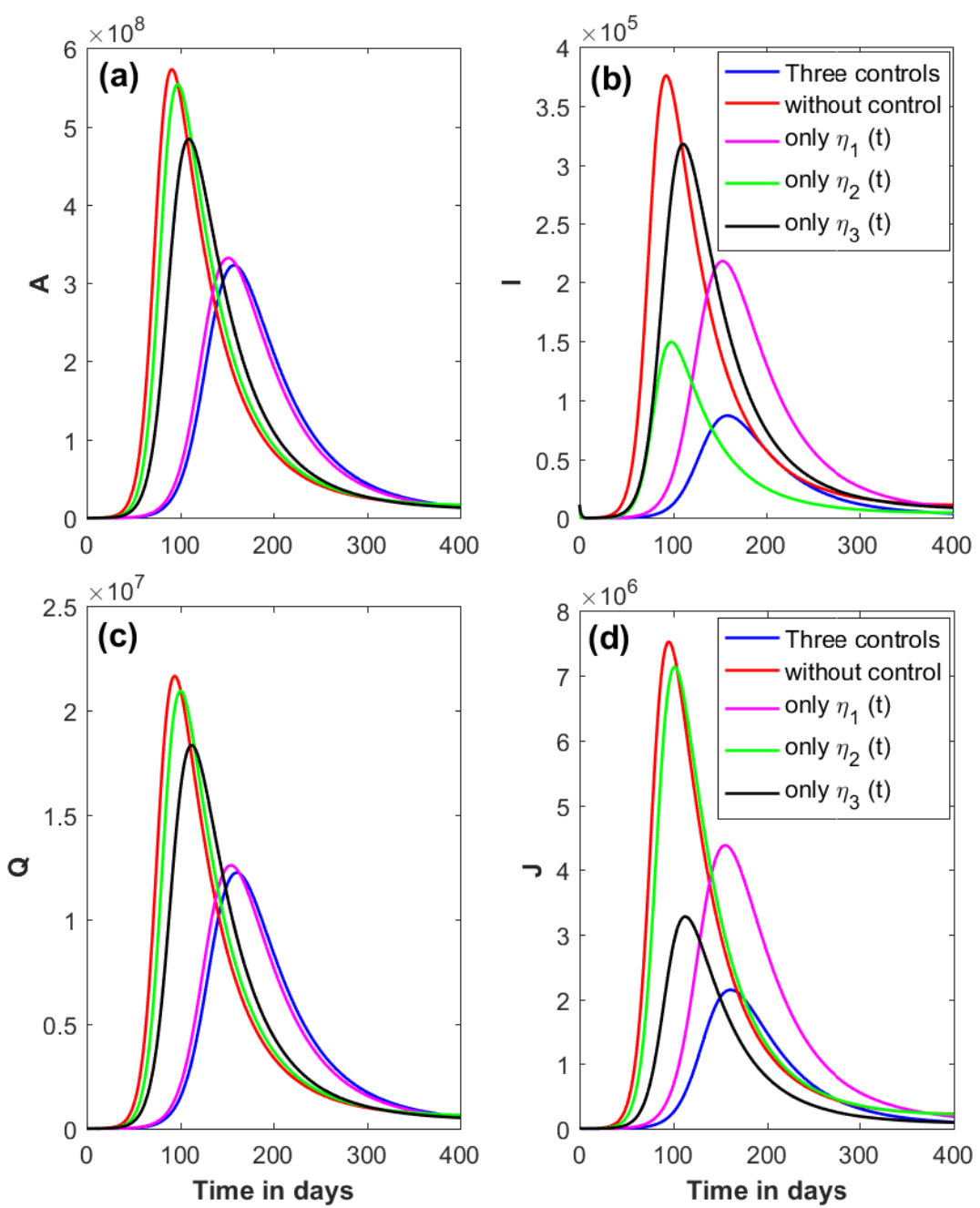

Fig. 8 The figure represents the comparisons of the corresponding to the (a) asymptomatic infected cases, (b) clinically ill or infected individuals, (c) quarantine individuals, (d) hospitalized or isolated individuals without intervention strategies, with the implementation of intervention strategies (combination of three controls and single controls). Optimal treatment strategy (solid blue line) demonstrates substantial reduction in all the state solutions when compared with the no controls and the application of single controls. The parameter values are given in the Table 2 when $R_{0}=1.8469$.

significant reduction of the asymptomatic cases, clinically ill cases, quarantine cases and isolated cases when compared with the no intervention scenario. The solid magenta curves, solid green curves and solid black curves represents the single controls namely $\eta_{1}(t), \eta_{2}(t)$ and $\eta_{2}(t)$, respectively shows the reduction of the asymptomatic cases, clinically ill cases, quarantine cases and isolated cases when compared with the no intervention strategies as well the implemen- 

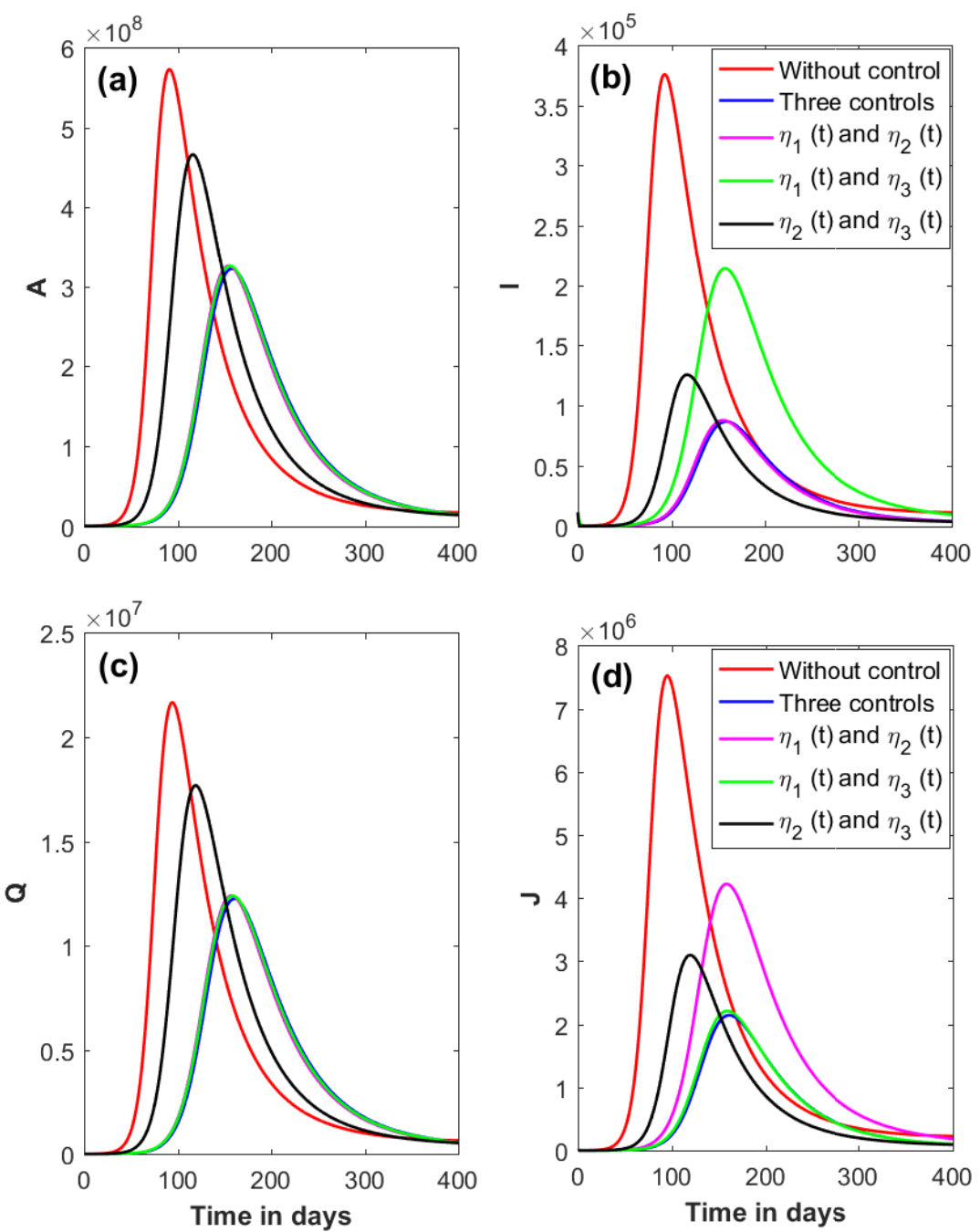

Fig. 9 The figure represents the comparisons of the corresponding to the (a) asymptomatic infected cases, (b) clinically ill or infected individuals, (c) quarantine individuals, (d) hospitalized or isolated individuals without intervention strategies, with the implementation of intervention strategies (combination of three controls and combination of two controls). Optimal treatment strategy (solid blue line) demonstrates substantial reduction of the cases in all the state solutions when compared with the no controls. The parameter values are given in the Table 2 when $R_{0}=1.8469$.

tation of three controls. Implementation of single control is better in compared with the without control policy. Also, in the cases for asymptomatic cases (only $\eta_{1}(t)$ ), clinically ill cases (only $\eta_{2}(t)$ ), quarantine cases (only $\eta_{1}(t)$ ) and isolated or hospitalized cases (only $\eta_{3}(t)$ ) shows the significant reduction of the individuals when compared with the no intervention scenario and compared 

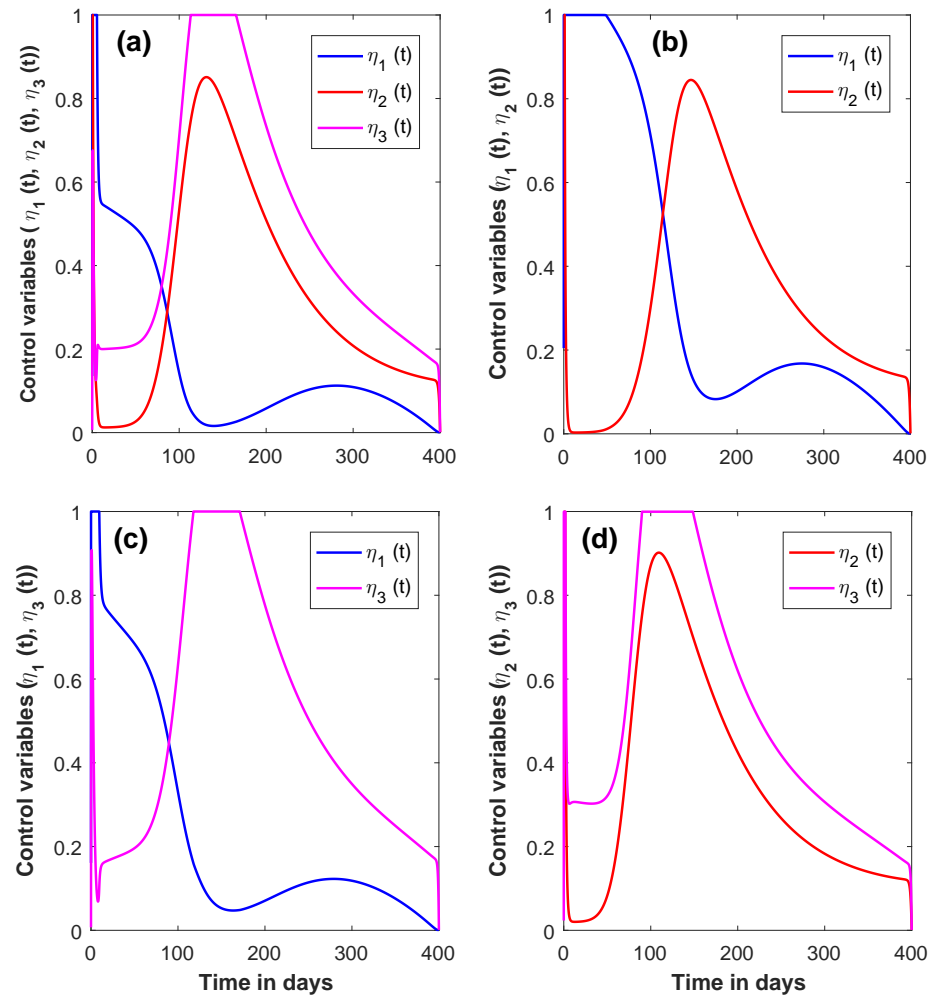

Fig. 10 The plot shows the optimal control functions as a function of time corresponding to the (a) three controls $\left(\eta_{1}(t), \eta_{2}(t)\right.$ and $\left.\eta_{3}(t)\right)$; (b) two controls $\left(\eta_{1}(t)\right.$ and $\left.\eta_{2}(t)\right)$; (c) two controls $\left(\eta_{1}(t)\right.$ and $\left.\eta_{3}(t)\right)$; (d) two controls $\left(\eta_{2}(t)\right.$ and $\left.\eta_{3}(t)\right)$. Parameters are given in the Table 2

with the implementation of three controls. Thus, from the Fig. $8((\mathrm{a})-(\mathrm{d}))$ we can conclude that the implementation of intervention strategy has an impact in controlling the transmission dynamics of novel coronavirus epidemic.

Now, we employ different treatment strategies for the disease compartments, namely asymptomatic individuals, symptomatic or clinically ill population, quarantined cases and the isolated individuals. The time series Fig. $9((a)-(d))$ represents the impact of each treatment strategies on the disease state variables in presence of the optimal control strategy and in absence of optimal treatment strategy for $R_{0}=1.8469$. These plots demonstrates the asymptomatic cases, daily number of infected cases, quarantine individuals and the isolated or hospitalized cases under no control $\left(\eta_{1}(t)=\eta_{2}(t)=\eta_{3}(t)=0\right)$, with the combination of three different controls $\left(\eta_{1}(t), \eta_{2}(t), \eta_{3}(t)\right)$ and the combination of two controls, namely $\eta_{1}(t)$ and $\eta_{2}(t) ; \eta_{1}(t)$ and $\eta_{3}(t) ; \eta_{2}(t)$ and $\eta_{3}(t)$. The solid red curves for epidemic (without implementation of intervention strategies) are shown to highlight the difference from those created via administration of optimal treatment polices. The solid blue epidemic curves 

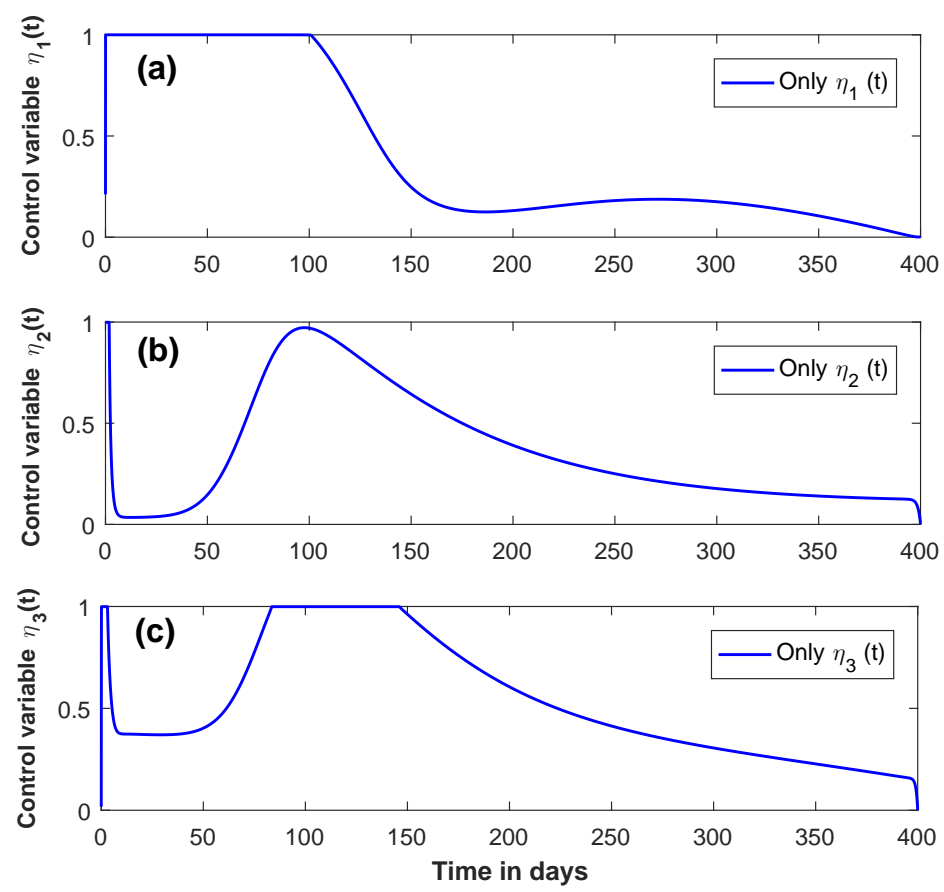

Fig. 11 The plot shows the optimal control functions as a function of time corresponding to the single control (a) $\eta_{1}(t)$, (b) $\eta_{2}(t)$ and (c) $\eta_{3}(t)$. Parameters are given in the Table 2

(with the administration of the combined of three controls) are exhibited the significant reduction of the asymptomatic cases, clinically ill cases, quarantine cases and isolated cases when compared with the no intervention strategies. The solid magenta curves, solid green curves and solid black curves denotes the combination of two controls namely $\eta_{1}(t)$ and $\eta_{2}(t) ; \eta_{1}(t)$ and $\eta_{3}(t) ; \eta_{2}(t)$ and $\eta_{3}(t)$, respectively shows the reduction of the asymptomatic cases, clinically ill cases, quarantine cases and isolated cases when compared with the no intervention strategies as well the implementation of three controls. Implementation of the combination of two controls is better when compared with the no control policy. Also, in the cases for asymptomatic cases (when $\eta_{1}(t)$ and $\eta_{3}(t)$ ), clinically ill cases (when $\eta_{1}(t)$ and $\eta_{2}(t)$ ), quarantine cases (when $\eta_{1}(t)$ and $\eta_{3}(t)$ ) and isolated or hospitalized cases (when $\eta_{1}(t)$ and $\eta_{3}(t)$ ) shows the significant reduction of the individuals when compared with the combination of three controls. Thus, from the numerical simulations (Fig. $9((\mathrm{a})-(\mathrm{d}))$ ) we can infer that the implementation of intervention strategy has an impact in controlling the transmission dynamics of novel coronavirus epidemic. Also, we can conclude that the combination of two controls are more effective when compared with the implementation of the single control. It can also be noted 
that combination of three controls is more influential when compared with the combination two controls as well as single controls.

The control functions $\left.\eta_{2}(t)\right)$ and $\eta_{3}(t)$ ) represents the antiviral treatment effectiveness aimed at clinically ill or infected cases and hospitalized or isolated populations, respectively. Another control function $\eta_{1}(t)$ ) maintains the mitigation of the creation of secondary infected cases resulting from the isolated individuals. The numerical results for the controls has been plotted in the Fig. 10 ((a)-(d)). The numerical solutions of the implementation of three control effectiveness simultaneously illustrated in the Fig. 10 (a). Corresponding to the implementation of the combinations of two controls, namely controls $\eta_{1}(t)$ ) and $\left.\left.\eta_{2}(t)\right) ; \eta_{1}(t)\right)$ and $\left.\left.\eta_{3}(t)\right) ; \eta_{2}(t)\right)$ and $\left.\eta_{3}(t)\right)$ has been shown in the Fig. 10 (b); Fig. 10 (c); Fig. 10 (d), respectively. The plot of the three graphs (see the Fig. 11) represent the optimal control functions where single control strategies, namely $\eta_{1}(t), \eta_{2}(t)$ and $\eta_{3}(t)$ has been implemented. The optimal control strategies based on single controls need the administration of in-depth effectiveness at the starting of an outbreak (Fig. 11(a)-(c)) of the novel coronavirus followed by quick reductions (most likely the outcome of high reduction in the levels of susceptible individuals).

\section{Discussion}

Reducing the influence and span of the ongoing novel coronavirus pandemic was on the intellects of every newsreader, Govt. official, public health departments, politicians and literally millions of populations throughout the world within a couple of months ago. According to the report by WHO, about 22 crore populations are infected with the novel coronavirus throughout the world 51. Though the Govt. of different countries and clinical persons of each and every country are trying to give safeguards to the individuals, still the rate of infection is high due to absence of proper vaccination for the COVID-19 virus. After the Second World War, the world has never gone through like the current situation that arrived due to novel coronavirus pandemic. Beginning from the Wuhan city of China in December 2019, the SARS-CoV-2 has outspread throughout the world. Due to absence of proper antiviral or effective treatment of novel coronavirus, one can rely on the qualitative control of novel coronavirus disease in spite of the elimination. In absence of proper vaccination and with an incomplete understanding of epidemiological cycle, mathematical models may help in understanding of both novel coronavirus transmission and its control.

To keep this pandemic scenario in our mind, we proposed and studied a compartmental SAIQJR model for novel coronavirus. Theoretical as well as numerical analysis of model system has been investigated using stability theory. Positivity and boundedness of the state equations assured that the proposed model (1) is well-defined. Our model system (1) possess two equilibrium points, namely a unique disease-free fixed point $E_{0}$ and a unique endemic equilibrium point $E^{*}$ whose feasibility and stability criteria rely on the numerical 
value of $R_{0}$. Using the theory of center manifold, we showed that the unique endemic steady state $E^{*}$ is locally asymptotically stable when basic reproduction number $R_{0}$ exceeds 1 . We also performed the global stability criteria for the disease-free fixed point $E_{0}$. The parameters in the Table 2 , we obtained the basic reproduction number $R_{0}=1.8469$ that demonstrates the significant outbreak of the SARS-CoV-2 virus.

We calibrated our proposed model based on the daily new novel coronavirus cases and cumulative number of novel coronavirus cases of India. Our proposed model have 14 parameters and we estimated only 6 parameters rely on the sensitivity analysis. As the sensitivity analysis exhibited that only 6 parameters are most sensitive with reference to the clinically ill or infected individuals. We also performed the normalized forward sensitivity analysis for our proposed model which reveals that the disease transmission rate $\beta_{s}$, recruitment rate $\Lambda_{s}$ of susceptible individuals, modification factor for asymptomatic individuals $\alpha_{a}$ and the mortality rate $\delta$ of all the individuals have an important effect on the clinically ill individuals and $R_{0}$. Our model illustration shows that to control the disease transmission individuals must have to maintain the social distancing, contact tracing, avoid mass gathering and use proper sanitization.

Moreover, we have modified our SAIQJR model by introducing optimal control problem. Implementation of intervention policies aids to mitigate the COVID-19 disease burden. To investigate the influence of non-pharmaceutical and pharmaceutical intervention policies and to stop the community transmission of the SARS-CoV-2 virus, we implemented three different control measures $\eta_{i}(t)$ for $i=1,2,3$. We established the existence of the optimal control functions. Moreover, to mitigate the infected populations and to minimize the cost of the controls, an objective functional $\mathcal{W}\left(\eta_{1}(t), \eta_{2}(t), \eta_{3}(t)\right)$ has been formed and interpreted with the aid of Pontryagin's Maximum Principle.

The behavioral changes in the susceptible individuals alters with time and to prevent or limit the rate of interaction between susceptible $(S)$ and isolated $(J)$ individuals, we put the external effort $\eta_{1}(t)$ an intervention strategy. Also the clinically ill or infected individuals become aware about the coronavirus disease fatality and consult with medical officers or admit to the hospitals or nursing home if the symptom occurs and thus we use the controlling effort $\eta_{2}(t)$ that changes the fraction of clinically ill cases. Again, we employ the controlling effort $\eta_{3}(t)$ that taking into account the cost regarding proper diagnosis, use of test kits and life-saving medicines that given to the hospitalized or isolated individuals. Thus, we considered all these three control strategies in our proposed model.

Finally, through extensive numerical simulations, we conclude certain observations. Numerical simulations shows in the Fig.11(a) that the intervention strategy presenting behavioral response $\eta_{1}(t)$ efforts with high magnitude immediately after introduction but slowly it reduces with time. Another way, the control strategy representing clinically ill or infected cases $\eta_{2}(t)$ efforts with its high magnitude for quite a long time and then it reduces (see the Fig. 11 (b)). Later on, the control indicating better treatment strategy for isolated 
individuals $\eta_{3}(t)$ efforts with high magnitude nearly about 3 months following a mitigation at a later stage, though this magnitude is higher than the magnitude of $\eta_{2}(t)$ (see the Fig. 11 (c)). From the extensive numerical simulations (see the Fig. 9((a)-(d))) we can infer that the implementation of intervention strategy has an impact in controlling the transmission dynamics of novel coronavirus epidemic. Also, we can conclude that the combination of two controls are more effective when compared with the implementation of the single control. It can also be noted that combination of three controls is more influential when compared with the combination two controls as well as single controls. Thus, implementing all the three control strategies may help to mitigate novel coronavirus disease transmission at this present epidemic scenario.

\section{Conflict of interest}

The authors declare that there is no conflict of interests regarding the publication of this article.

\section{Ethical standard}

The authors state that this research complies with ethical standards. This research does not involve either human participants or animals.

\section{Data availability statement}

All data generated or analyzed during this study are within the paper.

\section{Author contributions statement}

Jayanta Mondal and Subhas Khajanchi designed and performed the research as well as wrote the paper. The authors contributed equally to this work. All authors read and approved the final manuscript.

\section{References}

1. Britton, T., Ball, F., Trapman, P.: A mathematical model reveals the influence of population heterogeneity on herd immunity to SARS-CoV-2. Science 369(6505), 846$849(2020)$

2. Busenberg, S., Cooke, K.: Vertically transmitted diseases: models and dynamics. Vol 23. Springer Science \& Business Media, (1993)

3. Kermack, W.O., McKendrick, A.G.: A contribution to the mathematical theory of epidemics. Proc. Math. Phys. Eng. Sci. P Roy. Soc. A-Math. Phy. 115(772), 700-721 (1927)

4. May, R.M., Anderson, R.M.: Population biology of infectious diseases: Part II. Nature 280(5722), 455-461 (1979)

5. India covid-19 tracker. https://www.covid19india.org/ (2020) 
6. Ferguson, N., Laydon, D., Gilani, G. N., Imai, N., Ainslie, K., Baguelin, M., Bhatia, S., Boonyasiri, A., Perez, Z. C., Cuomo-Dannenburg, G., et al.: Report 9: Impact of non-pharmaceutical interventions (NPIs) to reduce covid-19 mortality and healthcare demand (2020)

7. Pecoraro, F., Clemente, F., Luzi, D.: The efficiency in the ordinary hospital bed management in Italy: An in-depth analysis of intensive care unit in the areas affected by COVID-19 before the outbreak. PLOS ONE 15(9), 1-15 (2020)

8. Flaxman, S., Mishra, S., Gandy, A., et al.: Estimating the effects of non-pharmaceutical interventions on COVID-19 in Europe. Nature 584(7820), 257-261 (2020)

9. Kantner, M., Koprucki, T.: Beyond just "flattening the curve": Optimal control of epidemics with purely non-pharmaceutical interventions. J. Math. Ind. 10(1), 23 (2020)

10. Giordano, G., et al.: Modelling the COVID-19 epidemic and implementation of population-wide interventions in Italy. Nat. Med. 26, 855-860 (2020)

11. Wu, J.T., Leung, K., Leung, G.M.: Nowcasting and forecasting the potential domestic and international spread of the $2019-\mathrm{nCoV}$ outbreak originating in Wuhan, China: a modelling study. LANCET. 395, 689-697 (2020)

12. Metcalf, C.J.E., Morris, D.H., Park, S.W.: Mathematical models to guide pandemic response. Science 369, 368-369 (2020)

13. Lopez, L., Rodo, X.: The end of social confinement and COVID-19 re-mergence risk. Nat. Hum. Behav. 4, 746-755 (2020)

14. Hoertel, N., et al.: A stochastic agent-based model of the SARS-CoV-2 epidemic in France. Nat. Med. 26, 1417-1421 (2020)

15. Kissler, S.M., Tedijanto, C., Goldstein, E., Grad, Y. H., Lipsitch, M.: Projecting the transmission dynamics of SARS-CoV-2 through the postpandemic period. Science 368, 860-868 (2020)

16. Nazarimehr, F., Pham, V.T., Kapitaniak, T.: Prediction of bifurcations by varying critical parameters of COVID-19. Nonlinear Dyn. 101(3), 1681-1692 (2020)

17. Weitz, J.S., Beckett, S.J., Coenen, A.R.: Modeling shield immunity to reduce COVID-19 epidemic spread. Nat. Med. 26(6), 849-854 (2020)

18. Ghosh, S., Senapati, A., Mishra, A., Chattopadhyay, J., Dana, S.K., Hens, C., Ghosh, D.: Reservoir computing on epidemic spreading: A case study on COVID-19 cases. Phys. Rev. E 104, 014308 (2021)

19. Nadim, Sk., Chattopadhyay, J.: Occurrence of backward bifurcation and prediction of disease transmission with imperfect lockdown: A case study on COVID-19. Chaos Soliton Fract. 140, 110163 (2020)

20. Mandal, M., Jana, S., Nandi, S.K., Khatua, A., Adak, S., Kar, T.K.: A model based study on the dynamics of COVID-19: Prediction and control. Chaos Soliton Fract. 136, 109889 (2020)

21. Das, P., Upadhyay, R.K., Misra, A.K., Rihan, F.A., Das, P., Ghosh, D.: Mathematical model of COVID-19 with comorbidity and controlling using non-pharmaceutical interventions and vaccination. Nonlinear Dyn. (2021) doi:10.1007/s11071-021-06517-w

22. Khajanchi, S., Sarkar, K., Mondal, J.: Dynamics of the COVID-19 pandemic in India. (2020) arXiv preprint arXiv:2005.06286

23. Khajanchi, S., Sarkar, K.: Forecasting the daily and cumulative number of cases for the COVID-19 pandemic in india. Chaos. 30, 071101 (2020)

24. Samui, P., Mondal, J., Khajanchi, S.: A mathematical model for COVID-19 transmission dynamics with a case study of India. Chaos Soliton Fract. 140, 110173 (2020)

25. Sarkar, K., Khajanchi, S., Nieto, J.J.: Modeling and forecasting of the COVID-19 pandemic in India. Chaos Soliton Fract. 139, 110049 (2020)

26. Khajanchi, S., Sarkar, K., Mondal, J., Nisar, K.S., Abdelwahab, S.F.: Mathematical modeling of the COVID-19 outbreak with intervention strategies. Results Phys. 25, 104285 (2021)

27. Rai, R.K., Khajanchi, S., Tiwari, P.K., Venturino, E., Misra, A.K.: Impact of social media advertisements on the transmission dynamics of COVID-19 pandemic in India. J. Appl. Math. Comput. (2021) DOI: 10.1007/s12190-021-01507-y.

28. Liu, X., Zheng, X., Balachandran, B.: COVID-19: data-driven dynamics, statistical and distributed delay models, and observations. Nonlinear Dyn. 101(3), 1527-1543 (2020) 
29. Khyar, O., Allali, K.: Global dynamics of a multi-strain SEIR epidemic model with general incidence rates: application to COVID-19 pandemic. Nonlinear Dyn. 102(1), 489$509(2020)$

30. Rohith, G., Devika, K.B.: Dynamics and control of COVID-19 pandemic with nonlinear incidence rates. Nonlinear Dyn. 101(3), 2013-2026 (2020)

31. Huang, J., Qi, G.: Effects of control measures on the dynamics of COVID-19 and doublepeak behavior in spain. Nonlinear Dyn. 101(3), 1889-1899 (2020)

32. Silva, C.J., Cruz, C., Torres, D.F.M., Munuzuri, A.P., Carballosa, A., Area, I., Nieto, J.J. et al.: Optimal control of the COVID-19 pandemic: controlled sanitary deconfinement in Portugal. Sci. Rep. 11, 3451 (2021)

33. Khajanchi, S.: Stability analysis of a mathematical model for glioma-immune interaction under optimal therapy. Int. J. Nonlinear Sci. Numer. Simul. 20(3-4), 269-285 (2019).

34. Khajanchi, S., Banerjee, S.: A strategy of optimal efficacy of T11 target structure in the treatment of brain tumor. J. Biol. Syst. 27(2), 225-255 (2019)

35. Khajanchi, S., Ghosh, D.: The combined effects of optimal control in cancer remission. Appl. Math. Comput. 271, 375-388 (2015)

36. Heesterbeek, H., Anderson, R.M., Andreasen, V., et al.: Modeling infectious disease dynamics in the complex landscape of global health. Science. 347, 6227 (2015)

37. Sharomi, O., Malik, T.: Optimal control in epidemiology. Ann. Oper. Res. 251(1-2), 55$71(2017)$

38. Rawson, T., Brewer, T., Veltcheva, D., Huntingford, C., Bonsall, M.B.: How and when to end the COVID-19 lockdown: An optimization approach. Front. Public Health. 8, $262(2020)$

39. Tsay, C., et al.: Modeling, state estimation, and optimal control for the US COVID-19 outbreak. Sci. Rep. 10, 10711 (2020)

40. Libotte, G.B., Lobato, F.S., Platt, G.M., Neto, A.J.S.: Determination of an optimal control strategy for vaccine administration in COVID-19 pandemic treatment. Comput. Methods Programs Biomed. 196, 105664 (2020)

41. Diekmann, O., Heesterbeek, J.A.P., Metz, J.A.J.: On the definition and the computation of the basic reproduction ratio $R_{0}$ in models for infectious diseases in heterogeneous populations. J. Math. Biol. 28(4), 365-382 (1990)

42. Driessche, P.V.D., Watmough, J.: Reproduction numbers and sub-threshold endemic equilibria for compartmental models of disease transmission. Math. Biosci. 180, 29$48(2002)$

43. Gantmacher, F.R.: The theory of matrices. Vol. 1, AMS Chelsea Publishing, Providence, RI, (1998).

44. Lienart, A., Chipart, H.: Sur le signe de la partie reelle des racines d'une equation algebrique. Journal de Mathematiques Pures et Appliquees (6 eme serie). 10, 291346 (1914)

45. Castillo-Chavez, C., Song, B.: Dynamical models of tuberculosis and their applications. Math. Biosci. Eng. 1(2), 361-404 (2004)

46. Pontryagin, L.S., Boltyanskii, V.G., Gamkrelidze, R.V., Mishchenko, E.F.: The Mathematical Theory of Optimal Processes. Wiley-Interscience, New York, (1962)

47. Schechter, M.: Principles of Functional Analysis. American Mathematical Society, Providence, RI (2001)

48. Kamien, M.I., Schwartz, N.L.: Dynamic Optimization: The Calculus of Variations and Optimal Control in Economics and Management. North-Holland, Amsterdam, (1991).

49. Lukes, D.L.: Differential Equations: Classical to Controlled. Math. Sci. Engrg. 162, Academic Press, New York, (1982).

50. Gumel, A.B., Ruan, S., Day, T., Watmough, J., Brauer, F., Driessche, P.V.D., Gabrielson, D., Bowman, C., Alexander, M.E., Ardal, S., Wu, J., Sahai, B.M.: Modelling strategies for controlling SARS outbreaks. Proc. R. Soc. Lond. B. 271, 2223-2232 (2004)

51. World Health Organization, Situation report. https://www.who.int/emergencies/diseases /novelcoronavirus-2019/situation-reports (2021).

52. Banerjee, S., Khajanchi, S., Chaudhuri, S.: A mathematical model to elucidate brain tumor abrogation by immunotherapy with T11 target structure. PLoS One. 10(5), e0123611 (2015), doi.org/10.1371/journal.pone.0123611

53. Fink, M.: myAD: fast automatic differentiation code in Matlab. (2006) http://www.mathw orks.com/matla bcent ral/filee xchan ge/loadF ile. do?objec $\mathrm{tId}=15235$. (Accessed 28 Oct 2016) 